\title{
Coups d'œil sur le Brésil Brazilian Survey
}

\section{I. - HISTORIQUE}

Un jour de mars de l'an 1500, partait de Lisbonne une flotte portugaise de 13 vaisseaux portant 1.500 hommes commandes par le jeune amiral Pedro Alvarez Cabral, dans le dessein avoué de refaire le parcours de Vasco de Gama conduisant aux Indes Orientales par le Cap de Bonne-Espérance. Fut-ce l'effet de vents contraires, ou plutôt celui d'une dérive volontaire impriméc à la flotte par Cabral, exécutant ainsi des ordres du roi Manuel, secrètement instruit depuis longtemps de l'existence d'un continent au sud de celui de Colomb? On ne le saura sans doute jamais, la destruction des archives de la monarchie lusitanienne par le tremblement de terre de Lisbonne ayant tari l'unique source où le monde pouvait trouver une réponse à la question : "Qui fut le premier à découvrir l'Amérique du Sud? "Quoi yu'il en soit, le 22 avril 1500, Cabral débarquait sur une terre inconnue, qu'il prit d'abord pour une île et dénomma aussitôt : Isla da Santa Cruz, gardant pour le point d'atterrissage le nom de Porto Seguro. Cahral, le 2 mai, poursuivait son voyage vers les Indes, en laissant sur place Gaspard de Lemos avec mission de remonter la cote vers le nord, puis de rapporter au Portugal la nouvelle de la découverte, ainsi que des échantillons de la faune et de la flore.

Obnubilé par les trésors du pays de Golconde, le Portugal, libéré depuis deux siècles seulement de la domination arabe et encore dépourvu d'hommes et d'argent liquide, s'intéresse peu à la découverte du nouveau pays, dont il ignore encore que ce n'est là qu'une partie d'un immense continent; les "Lusiades " de Camoëns n'ont pas un mot pour cette contréc qui, cependant, trois siècles plus tard, sauvera le Portugal et sá monarchie. Méprisée reste l'Isli da Santa Cruz, vite devenue dédaigneusement la "Tlerra dos Papagaios ", jusqu'au deuxième voyage de découverte (1531), décelant l'abondance d'un précieux bois tinctorial, le « pão vermelho " out «pâu brazil » (cle " brazo »: luire, briller comme braise - parce que la section de ce bois a des reflets rouges brillants), (jui finira par fairo donner le nom de Brazil a tout le pays.

Cependant, l'Espagne, impérialiste par vocation, s'y intéresse dangereusement pour son voisin, et il ne faut rien moins que l'application des clauses du providentiel traité de 'lordesillas -- intervenu en 1494 entre les deux nations pour définir les périmetres d'influence respectifs - pour que, en 1506, le Portugal se voie assuré d'une possession paisible du Brésil.

A partir de 1530 , la colonisation est abandonnéc a l'initiative privée: dès 1534 , une quinzaine de « capitanias » se partagent les côtes du pays, chacune d'elles étant administrée par un homme qui a reça du roi l'entière propriété avec ses conséquences : transmissibilite par hoirie ou par cession, souveraineté. Mais, deux d'entre elles mises à part: celle de São Vicente et celle de la Nova Iusitania (l'actuel Pernambuco), que la culture de la canne ì sucre sortira de l'ornière, les autres capitaineries, mal gérées, périclitent, et menacent de devenir une 


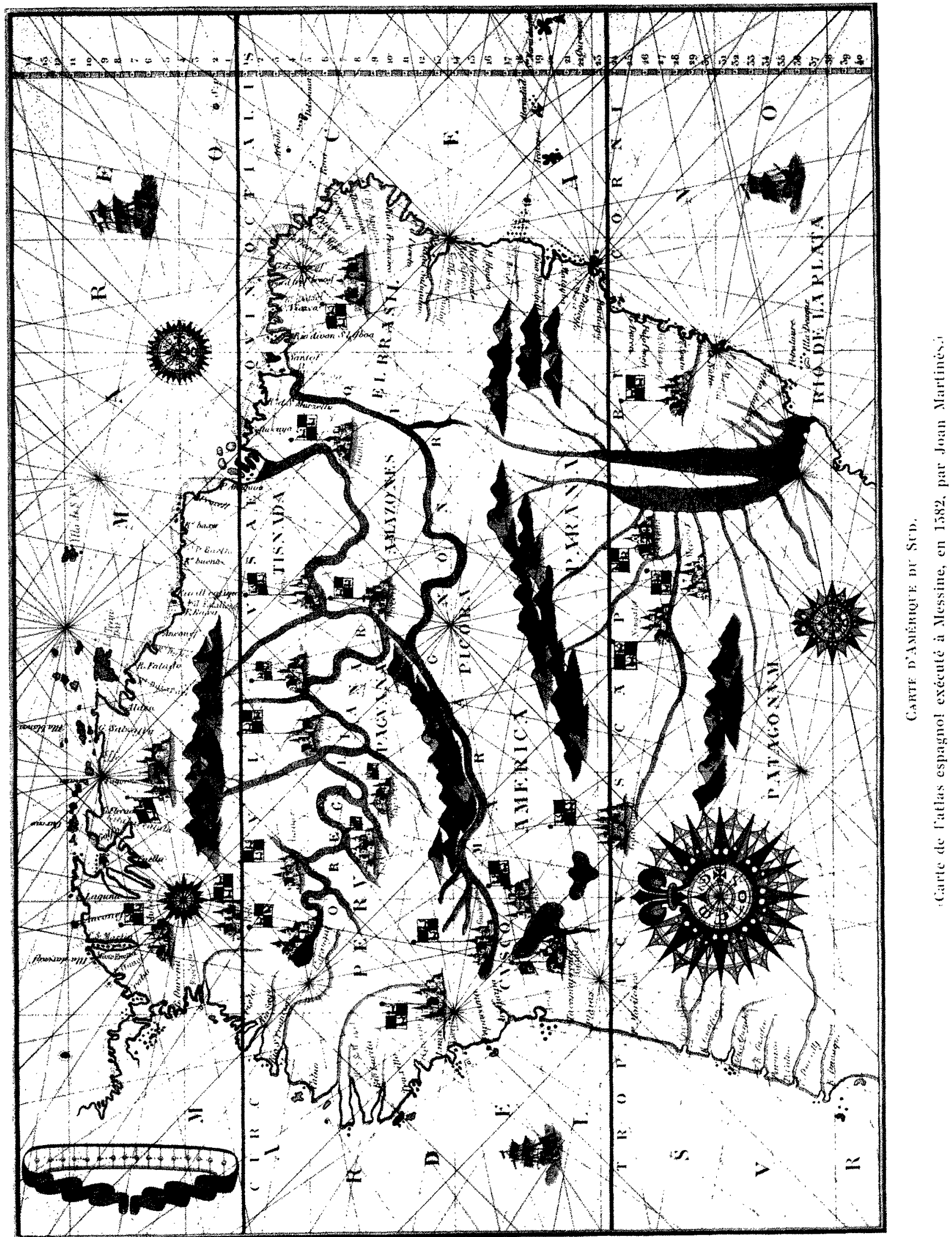


proie tentante pour les amatems de coups de force.

Aussi, en février 1549 , le roi João III, alerté par Luis de Goes dès le 12 mai précédent, envoie commc gonverneur général, avec mission d'organiser une capitale et une administration plus centralisée, Tomé de Souza, dont il avait pu apprécier le comportement dans les possessions d'Afrique et de l'Inde. Tomé de Souza, escorté de 600 soldats et de 100 " desgregados " (hors-la-loi, dévoyés), fortifie Bahia et instaure un gouvernement, puissamment aidé en cela par sept Pères Jésuites, ayant à leur lête Nanuel de Nobrega, qui l'avaient accompagné et dont l'ouvre, "sans exemple dans l'histoire", fut capitale pour la colonisation du pays et pour son unification lu point de rue des races, des limgnes, de la religion, des idées et des buts.

L'amorce d'une activite économique dans $f^{2}$ "Pays des Perroquels " ne pouvait manquer d'attirer l'attention et d'exciter l'avidité des autres nations. Et le 10 novembre 1555 , wne petite flotte française débarque, sur une des îles de la baie de Guanabara (près du Rio actuel), quelques centaines d'hommes cue commande Nicolas Durand de Villegaignon, chevalier de Rhodes, " mi-savant, mi pirate ", rêvant de fonder une "France Antarctique " dont il serait le maître, qui batit sur l'ile le fort Coligny et aménage un port pour les navires de commerce francais, lesquels se mettent à le friquenter. Cette petite enclave française subsista jusfu'en 1559: Ia présence a Bahia d'un nouveau gouverneur, Mem de Sí, et les exhortations de Manuel de Nobrega, déclenchent l'envoi par Lisbonne d'une expédition; celle-ci, après quelques mois passẻs en préparation, attaque et prend le fort Coligny, puis, sans poursuivre ses avantages, se retire vers sos bases sur le continent. Et le statu quo subsiste encore durant cinc années, au bout desquelles la reine slu Portugal, prêtant enfin l'oreille aux objurgations de Nobrega, envoie de Lisbonne Estacio de Sà ef les renforts; après dix mois de préparatifs, la nouvelle forteresse frangaise, le Morro de Gloria, esl cmportée et, sur ses ruines, l'évêque consacre une iglise qui sera la première de la future capitale: Rio de Janeiro. Les Francais se retirent sur leurs quatre vaisseaux, ne rapportant en France que le prtun dédić i Jean Nicot (1567)...

Peu à peu, les frontières du Brésil reculent, cependant que le roi Sébastien, dernier représentant de l'héroïque dynastie d'Aviz (branche cadette de la maison de Bourgogne), laquelle régnait depuis Joäo Ior, en 1380, succombe en 1578 à Alcacer-Quivir, en combattant les Maures; l'Espagne en profite pour s'emparer du trône vacant et, jusqu'en 1640 , le Portugal et ses colonies, dont le Brésil, deviennent espagnols.

Cela raut au Brésil lattention des ennemis de l'Espagne: les pirates anglais pillent Santos, brûlent São Vicente; les Francais s'installent en 1560 dans 1e " Maragnan " (l'actuel Maranhâo) et y bâtissent Saint-Louis (São Luiz), ainsi nommé en l'honneur du jeune roi Louis XIII. Ils s'y maintiendront jusqu'en 1615. Chose plus grave, les Hollandais pénètrent a Bahia, y confisquent la flotte, pillent les ports et commencent à conquérir " het'Zuickerland ": le pays du sucre. En 1621, ils fondent a Amsterdam la Compagnie des Indes Occidentales, à un capital considerable, en réplique a la Compagnie des Indes Orientales. Cette compagnie arme 26 navires avec 500 canons, 1.600 marins et 1.700 soldats de métier; le résultat désiré est promptement obtenu: le 9 mai 1624, Bahia est enlevée i nouveau, avec un immense butin. L'Espagne, secouée par l'annonce du désastre, expédie 50 navires et 11.000 hommes, qui reprennent Bahia. Mais les Hollandais envoient de nouveaux renforts : en 1635, Fernambuco (l'actuel Recife) est occupé, puis, très ientement, toute la côte nord-est jusqu’à Bahia. Ii jusqu'en 1644, la colonisation hollandaise mettra en valeur cette côte du Brésil, placée depuis 1637 sous la sage administration de Maurice de NassauSiegen, en l'honneur de gui Fernambuco devinl Mauritsstaad. En toute objectivité, on doit reconnaître que l'occupation par les Hollandais, qui se montrèrent organisateurs avisés et méthodiques, libé raux et plus evolués que les Portugais, fut bénéfique? pour le Bresil.

Pendant ce temps, en 1640, le Portugal avait retrouvé son autonomic, ses colonies et un roi, João IV. Par suite, l'intervention hollandaise au Brésil, fondéc juridiquement sur la lutte contre l'Espagne, devenait injustifié a partir du moment wi l'Espagne cessait de détenir en partie le Brésil : des pourparlers s'engagent, qui traineront en lonsileur juscuu'en 1649, où le P. Vieira fonde à Lis. bonne la Companhia Geral de Commercio para o Brasil, antagoniste de la compagnie hollandaise of qui équipe: au Portugal, une flotte, et au Brésil, une armée; tant at si bien que pendant que les pourparlers s'éternisent, la susdite armée s'impaliente et entre en lice; les Hollandais sont peu à peu repoussés et finissent par quitter définitivement le Brésil en 1658, après la capitulation de Mauritsstaad le 26 janvier 1654 .

Délivré de tout contròle etranger a celui du Portugal, le Brésil se découvre pen ì peu à lui-même. En même temps s'est formé lentement un type ethnicque nouveau, le "Brésilien ", Portugais ou fils de Portugais, en général vivant plutôt dans l'hinterland que sur les côtes, et dont le type le plus remarquable est le Pauliste, au caractère aventureux et par ailleurs peu riche de scrupules. Ces colons de São Paulo, cherchant l'aventurc, organisent périodiquement des raids de "bandeirantes » (bandes) opérant, dans les contrées voisines, des " entradas $\gg$ (incursions) au cours desquelles ils explorent, certes, découvrent parfois, dévastent souvent, pillent toujours et pratiquent à l'encontre des indigènes la chasse à l'homme et l'esclavage les plus odieux.

Ils découvrent l'or, puis le diamant, et pendant cinquante ans, c'est la ruée vers le Rio das Velhas, la croissance rapide de Villa Rica, de Villa Real, de Villa Albuquerque, jusqu'au tarissement total des gisements qui fit rentrer dans le néant tout cela, oi cependant subsistaient les voies de pénétration. C'est aussi la mainmise de l'autorité royalc sur l'économie du pays, et une réglementation draconienne, qui se perpétuent pendant plus d'un siècle et indisposent les "Brésiliens ». Ceux-ci suivent d'un cil intéressé les efforts de l'Amérique du Nord pour s'affranchir de la tutelle anglaise, et des conjurations, telles que l' "Inconfidencia Mineira " (ei 1788, dans les Minas Geraes) prennent naissance, 
qui auraient peut-être abouti à une sécession brutale entre la mère-patrie et sa colonie, si l'exode soudain de la monarchie n'avait changé pour un lemps le comportement gouvernemental si détesté.

Les péripéties de la guerre napoléonienne contre l'Angleterre, amenant la bataille jusque sur le terriloire portugais, mettent le régent João dans une alternative cruciale causée par le blocus continental : ou bien se refuser au blocus, irriter Napoléon et perdre le Portugal et son trône; ou mécontenter l'Angleterre, s'exposer au blocus maritime et perdre le Brésil. Le régent, se rendant compte enfin que la possession du Brésil risquait fort d'être plus sûre, plus fructueuse et même plus honorifique que celle du Portugal, lorsqu'il recoit, en novembre 1807, l'ultimatum napoléonien le sommant d'opter, n'attend pas larrivée du général Junot, le nouveau duc d'Abrantès; il se décide à suivre le conseil énoncé par João IV dans son testament: "Se réfugier au Brésil en cas de danger "; il s'embarque rapidement avec 15.000 personnes: noblesse, magistrature, clerge, chefs militaires, et aussi un viatique de 200 millions de cruzados; puis il vogue vers Bahia, sous la protection des vaisseaux de guerre anglais.

Un bouleversement dans les mours administralives du Brésil s'ensuit: infiniment plus de libertés dans tous les domaines, création d'une séric d'institutions uni èlèvent le Brésil au rang d'un royaume ef Rio de Janeiro au rang de capitale; puis, en 1815, l'égalité constitutionnelle des royaumes unis du Portugal et du Brésil est décrétée, en sorte que sans désordres ni sacrifices, le Brésil obtient ce (lu'une guerre d'indépendance ne lui aurait pas acquis à si bon compte. En 1816, la mort de la reinc Doña Maria Ire place sur le trône le régent, qui prend le nom de João VI.

Le Brésil se préoccupe alors tle consolider ses frontières, singulièrement élargies depuis qu'en 1750, sous Joseph $I^{\text {er }}$, la nullité des limites prévues par le traité de Tordesillas a été admise par le traité de Madrid. L'Uruguay tente João VI, qui l'annexe. La situation du royaume brésilien s'annonce comme si prospère que le fils de João VI, Dom Pedro, obtient sans difficulté de l'empereur' d'Autriche, François $I^{\text {cr }}$, la main de sa fille, la princesse Léopoldine, sceur de l'ex-impératrice Mariel.ouise.

Mais la paix etant rétablie en Europe depuis 1815 , le Portugal réclame la présence de son roi. Celui-ci, séduit par la beauté de son grand domaine, le Brésil, hésite pendant cinq ans à le quitter. Enfin, en 1820 , il nomme à sa place son fils Pedro et regagne à regret le Portugal.
Les treize années pendant lesquelles l'exode de la monarchic avait en quelque sorte interverti les rôles entre la colonic et la métropole, avaient marqué le Brésil d'une empreinte trop profonde et $y$ avaient éveillé trop d'aspirations pour que le Brésil pût se résoudre à reprendre le rang d'une possession coloniale. Le départ de Dom João VI ravive les vellèitès d'indépendance du peuple brésilien, velléités qu'encourage in petto, avec un opportunisme clairvoyant, le jeune et ambitieux Dom Pedro, installe par son père en qualité de "Defensor perpetuo do Brazil s, avec le conseil do se couronner luimême roi du Brésil si les circonstanees l'exigent. Après une résistance plus symbolique que réelle, le jeune "Defensor" soutenu par José Bonifario de Andrada e Silva -... futur remarquable homme d'Etat -, proclame a Ipiranga, le 7 septembre 1822, l'indépendance du Brésil à l'égard du Portugal, et le 12 octobre suivant, ceint la couronne d'cmpercur constitutionnel du Brésil, sous le nom de Pedro $1^{\text {"r. }}$.

Cependant, rêveur et maladroit, débauché et dominé jusque dans les affaires de l'Etat par sa maîtresse, la marquise de Sanlos, il ne sait ni gagner ni conserver l'affection de son peuple. Ia guerre malheureuse contre l'Argentine, détachant en 1828 l'Uruguay du nouvel empire, au grand mecontentement des Bresiliens, altaches à la possession de la province "cisplatina ", accroit leur ressentiment et, bien que Pedro $1^{\text {er }}$ ait, a la mort de son père, en 1830, renoncé à la couronne du Portugal, optant ainsi clairement pour le Brésil, l'empereur. conscient que le fait d'otre né au Portugal ne le consacrait pas comme un viai Brésilien, percevant aussi une sorte de choc en retour de la revolution francaise de juillet, abdique le 7 avril $1831 \mathrm{cl}$ faveur de son fils, Dom Pedro de Alcantara, igé de cing ans, et il quitte paisiblement le pays.

Après une régence mouvementéc, pedro II, né Brésilien, lui, est déclaré majewr à quinze ans, lo 18 juillet 1840. Il régnem paisiblement jusqu'en 1889. Apres avoir eu la grande joie de voir réalise, en 1888, le désir de toute sa vie: l'abolition definitive do l'esclavage au Brésil, mais n'ayant plus de fils vivant, ne pouvant songer at lasser la couronne à son gendre, un prince d'Eu, de la mai. son d'Orléans, il n'oppose ancune résistance à !a première tentalive de coup d'Etat: digne at silen. cieux, Dom Pedro II, au terme d'un règne d'un demi-siècle, quitle pour toujours le Brésil le 17 novembre 1889 , comme son père et son aïeul, emportant, sinon les regrets, du moins lestime le soil peuple.

Depuis, l'empire a fait place a la République fédérative des Etats-Unis du Brésil.

\section{II. - GÉOGRAPHIE - ETHNOLOGIE - SOCIOLOGIE}

\section{GÉOGRAPHE}

Le Brésil oceupe sensiblement la moitié de la surface de l'Amérique du Sud, avec environ $8.769 .000 \mathrm{~km}^{2}$, ce qui le place au troisieme rang des lerritoires nationaux dans le monde, apres l'U.P.S.S. et le Canada.
II s'ètend, en longitude onest, sur $39^{\prime \prime} 14^{\prime}$, entre les $37^{\circ}$ et $76^{\mathrm{e}}$ degrés, ce qui équivaut a environ $4.360 \mathrm{~km}$ et ce qui représente un décalage d'environ deux heures et demie entre les passages du soleil aux deux méridiens précités; en latitude, de $5^{\circ} 16^{\prime}$ de latitude nord (correspondant à celle d'Abidjan, en Côte-d'Ivoire), ì 33" 45' de latitude sud (corres- 
pondant a celle de Cape Town, en Afrique du Sud), donc sur $39^{\circ} 1^{\prime}$ au total, equivalant a plus de $4.300 \mathrm{~km}$; le Brésil est ainsi traversé par l'équateur, au niveau de Macapa, en Guyane brésilienne, dans la boucle du nord de l'Amazone, et par le tropique du Cancer, à 22 $30^{\circ}$ au sud, au niveau de São Paulo. C'est dire l'extrême diversité des climats, de la flore et de la faune qu'on est exposé à rencontrer en le parcourant du nord au sud.

Le développement des côtes représente près de $9.000 \mathrm{~km}$.

\section{Orographe}

L'orographie du Brésil est caractérisée par :

$1^{\circ}$ La plaine cotière atlantique, souvent fort etroite;

2" Une série de chaînes cotières, comprenant, du sud au nord, les serras Giral, do Mar, do Parandpiacaba, do Mantiquara $(2.712 \mathrm{~m})$, das Aymores et do Sincora;

3" Un immense platean adossé à celte série de chaines, fort tourmenté lui-même, semé de chaìnes parallèles ou non $\dot{a}$ la côte et culminant vers $1.950 \mathrm{~m}$, mais qui s'abaisse jusqu'à $300 \mathrm{~m}$ à l'ouest, au platean boisé du Matto Grosso;

4" Un groupe montagneux d'altitude moyenne, couvrant le nord-est du pays au nord du rio São Francisco, et coupé de nombreuses vallées orientées dans tous les sens;

5" A l'ouest et au nord, l'immense plaine boisce parcourue par l'Amazone et ses affluents.

Les vallées des rios Araguaya et Tocantins, et une partie de celle du rio São Francisco, sont aussi de larges plaines de basse altitude.

Ia présence des chaînes côtières très proches de l'Océan, et leur doublure par les sierras du plateat, ont formé et formeront longtemps un obstacle considérable à une facile pénétration dans l'hinterland.

\section{HYDROLOGIE}

Le réseau fluvial a pour principaux eléments : au nord, les rios São Francisco $(3.161 \mathrm{~km})$, Tocantins $(2.600 \mathrm{~km})$, Araguaya $(2.200 \mathrm{~km})$, Xingu $(2.000 \mathrm{~km})$ et Tapajoz $(2.000 \mathrm{~km})$; au sud, les rios Parana $(3.300 \mathrm{~km})$ et Paraguay $(2.206 \mathrm{~km})$; il est oriente dans le sens nord-sud ou vice versa et n'apporte ainsi aucune facilité à la pénétration dans le sens est-ouest, cependant la plus désirable. Seule, l'Amazone $(6.000 \mathrm{~km})$ et certains de ses affluents, la Maleira $(1.450 \mathrm{~km})$ et l'Yapura $(2.800 \mathrm{~km})$, navigables sur des distances allant de 1.200 a $1.900 \mathrm{~km}$, permettent cette pénétration même aux vaisseaux maritimes.

\section{GÉorogit:}

Considéré sous l'aspect géologique, le Brésil est constitue principalement par une assise cristalline de granit et de gneiss très ancienne, couvrant plus du tiers du territoire, bordée, sur l'intérieur du pays, de terrains sédimentaires gréseux, passant asse\% régulièrement du système primaire au sys- teme tertiaire, lequel est le plus représente au fur et à mesure que l'on s'enfonce du sud au nord. Les formations montagneuses, en modifiant cette structure de base, ont donné naissance à un sous-sol peu riche, ou l'on rencontre des gisements de minerais métalliques (parfois extrêmement importants) et un peu de mauvais charbon, et $\dot{a}$ des sols arides pauvres en chaux et en phosphore.

\section{Cumat}

Du point de vue climatologique, on distingue trois zones :

- La zone équatoriale, comprenant le nord et le nord-est du Brésil : température moyenne supérieure $\dot{a}+25^{\circ} \mathrm{C}\left(27^{\circ} \dot{\mathrm{a}}\right.$ Manaos), et $\dot{\mathrm{a}}$ oscillations thermiques faibles. La pluviosite y dessine trois types :

- type isosuperhumide, a précipitations annuclles superieures it $1.900 \mathrm{~mm}$ sans période sèche (nord-onest de l'Amazone);

- type superhumide, à pluviosité plus faible ef avec période sèche (nord-est de l'Amazone);

- type semi-aricie, a pluviosité inférieure à $600 \mathrm{~mm}$ et longnes périodes sèches (Para ef centre des Etats du Nord-Est).

- La zone tropicale, la plus vaste, de température comprise entre $20^{\circ}$ et $35^{\circ}$, avec des oscillations marquées (en moyenne, $23^{\circ} \mathrm{C}$ ); pluviosité de 1.300 a $1.900 \mathrm{~mm}$, surtout durant les mois d'été (premier trimestre de l'année).

- La zone tempérée, au sud. Températuro moyenne inférieure $\dot{a}+20^{\circ}$, saisons plus marquées: pluviosité de $1.900 \mathrm{~mm}$ sur la cote et de $1.300 \mathrm{~mm}$ clans l'hinterland, avec une période sèche en hiver (juin-aoùt) dans les parties montagneuses et accidentées du nord du Parana, du São Francisco ef du sud de Minas Geraes.

Les ecarts de temperature peuvent être très wrands: dans le Goias, dans la zone des sommets les plus élevés, le thermomètre peut marquer $+59^{\circ} \mathrm{C}$ au soleil a 14 heures et $+9^{\circ}$ la nuit suivante, soit $50^{\circ}$ de différence en quelques heures; c'est dire que pour les êtres humains vivant dans cette région, la température de $+9^{\circ}$ est aussi pénible que celle de $-15^{\circ}$ en Furope occidentale.

On a observé qu'à latitude égale, il fait beaucoup) moins chaud et le soleil se supporte mieux qu'en Europe ou en Afrique. Ainsi, a Recife, beaucoup de blancs sortent uu-tête au soleil de midi, alors qu'à Loando, en Angola, et à la mème latitude, ils seraient rapidement foudroyés par l'insolation. Rio de Janeiro, sous le tropique du Capricorne, est habitable l'été, alors que Roodebank, sous le mêne tropique, mais dans le sud-ouest africain, est torride, et que Villa Cisneros, en Rio de Oro, sous le tropique du Cancer, est une fournaise plus de six mois par an. I'hiver, à latitude égale, il fait plus froid an Brésil qu'en Europe occidentale.

Dans l'ensemble, le climat est chaud et humide: il est assez salubre et supportable, sauf dans le bassin de l'Amazone. Mais le caractère d'uniformiti 
qu'il présente en diversesrégions est débilitant, et peu propice à l'effort humain.

Le sol lui-même subit l'érosion des pluies tropicales, et aussi le phénomène de latérisation; notons qu'il faut se garder de confondre la latérite arec la \& terra vermelha", qui est fertile.

Ce climat est, par contre, propice aux productions végetales; les forêts tropicales sont exubi rantes et dine diversité de production extraordinaire (on a identifié plus de 4.000 especes foreslières, contre 200 seulement en Europe); la péné. tration $y$ est d'ailleurs fort ardue, souvent impossible avec les moyens actuels : une grande partio du Matto Grosso (au nord du plateau de ce nom), dit Goias (surtout entre les rios Araguoya et Tocantins, au nord), de la Guyane brésilienne, et des "selvas " de l'Amazonie, est encore totalement inconnue.

\section{EThNologie}

La population est actuellement voisine de 56 millions l'âmes, évidemment réparties fort inegalement : la densité au $\mathrm{km}^{2}$ est d'environ 21 dans les Etats du Sud, et de 0,5 seulement dans ceux du Nord; en moyenne, 6,25 au $\mathrm{km}^{2}$. On peut dire que la zone côtière atlantique et quelques points de l'hinterland, totalisant le tiers environ de la superficie totale, abritent $95 \%$ de la population du Brésil.

C'est une population d'âge moyen assez bas; la durée moyenne de la vie est de quarante ans.

Parmi les éléments de cette population, on remarque, par ordre d'ancienneté :

-- Les aborigènes, Indiens de tribus diverses, surtout Tupis ef Tarnoios, progressivement refoulés dans les régions les moins accessibles (Matto Grosso, Amazonie, Goias) et en voie de diminution incessante; on en evalue le nombre entre 1,5 millions et 3 millions; a part les Chavantes et quelques ilots de Gayapos, toutes ces tribus sont pacifiées, mais elles onf conservé leurs habitudes nomades ef leurs mours et vivent en marge de la civilisation européenne.

- Les blanes, d'abord les Portugais, inslalles dans le pays depuis la découverte, ensuite accrus d'apports espagnols, français, hollandais, puis, après l'abolition de l'esclavage, par l'immigration italienne, allemande et slave; toutes les nations européennes sont plus ou moins représentées également; l'élémenl latin est dominant, et particulièrement le portugais, qui a donné au pays la langue, la religion et les mours. l'élément blanc réside surtout dans la zone tempéré.

-.- Les noirs, importés en masses considerables: les Soudanais, de la Guiné portugaise, les Bantous, des possessions portugaises de l'Angola et du Mozambique; ils prédominent surtout alu nord du $15^{\circ}$ parallele.

- Les asiatiques, surtout Japonais, venus lors de limmigration consécutive a l'abolition de l'esclavage et au demeurant parfaitement imperméables a la brésilianisation; aussi, quelques Hindous et quelques Chinois. guer :
- les mamelucos, isspus du croisement des blancs et des aborigenes;

-- les mulatres, issus du croisement des blanes et des négres;

-.- les zambos ou caboclos (litlealement " cuivrès "), issus du croisement des indicns et des neigres:

distinction an demeurant de phus en phus difficile, vu les multiples sur-croisements intervenus depuis longtemps, de sorte qu'buclydes da Cunha a pu dire, dans les "Sertôes » : "Naô ha um typo anthropologico brasileiro $\gg$ (il n'y a pas de "race brésilienne "); les immigrés de fraiche date mis i part, ainsi que quelques rares éléments de l'élite et de la noblesse brésilienne, la majorite du peuple brésilien représente un mélange d'une complexité inextricable. L'absence de ségrégation raciale et de préjugés de cet ordre chez l'élément portugais explique cette complexite et l'importance du métissage. On estime que la répartition est voisine de la suivante :

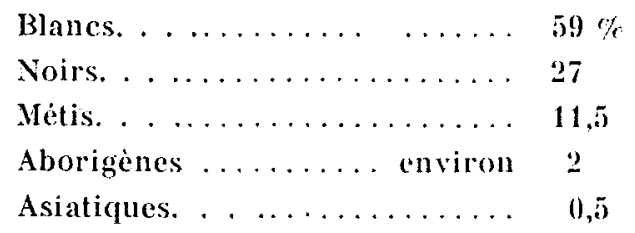

La natalité atteint le taux de 40 rí ef la mortalite générale celui de $20 \%$; l'un et l'autre sont parmi les plus élevés du monde. Le nombre des femmes tend insensiblement a l'emporter sur reluj des hommes. Ia répartition par áges accuse les chilfres suivants :

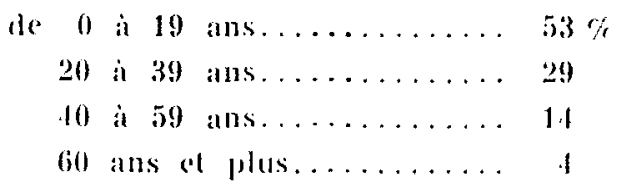

on est donc en face d'une population "jeune ". On considère que, dı fait du climat, l'Européen immigré vit cing ans de moins qu'il n'eût vécu en Europe; son fils, le crécle. dix ans, et son pelit-fils, quinze ans de moins.

le taux eleve de natalite, joint aux apports l'immigration, fait que l'aceroissement annuel, prisentement d'un million d'ames environ par an, irat en s'élevant promptement; les Brésiliens escomplent li: chiffre de 200 millions pour la population vers la fin du siecle présent. I.a capacité de peuplement, qui varie, suivant les evaluateurs, entre 100 et 850 millions, semble devoir etre plus vatisemblable. ment de 500 millions.

On estime que la population est repartic sensiblement de la facon stivinte:

Population urbaine . . . . . . . . . $25 \%$

Population suburbaine ef centres muratux de

plus de 1000 habitants. . . . . .

Population rurale $\ldots \ldots \ldots \ldots \ldots \ldots \ldots \ldots$ 6.3,5 


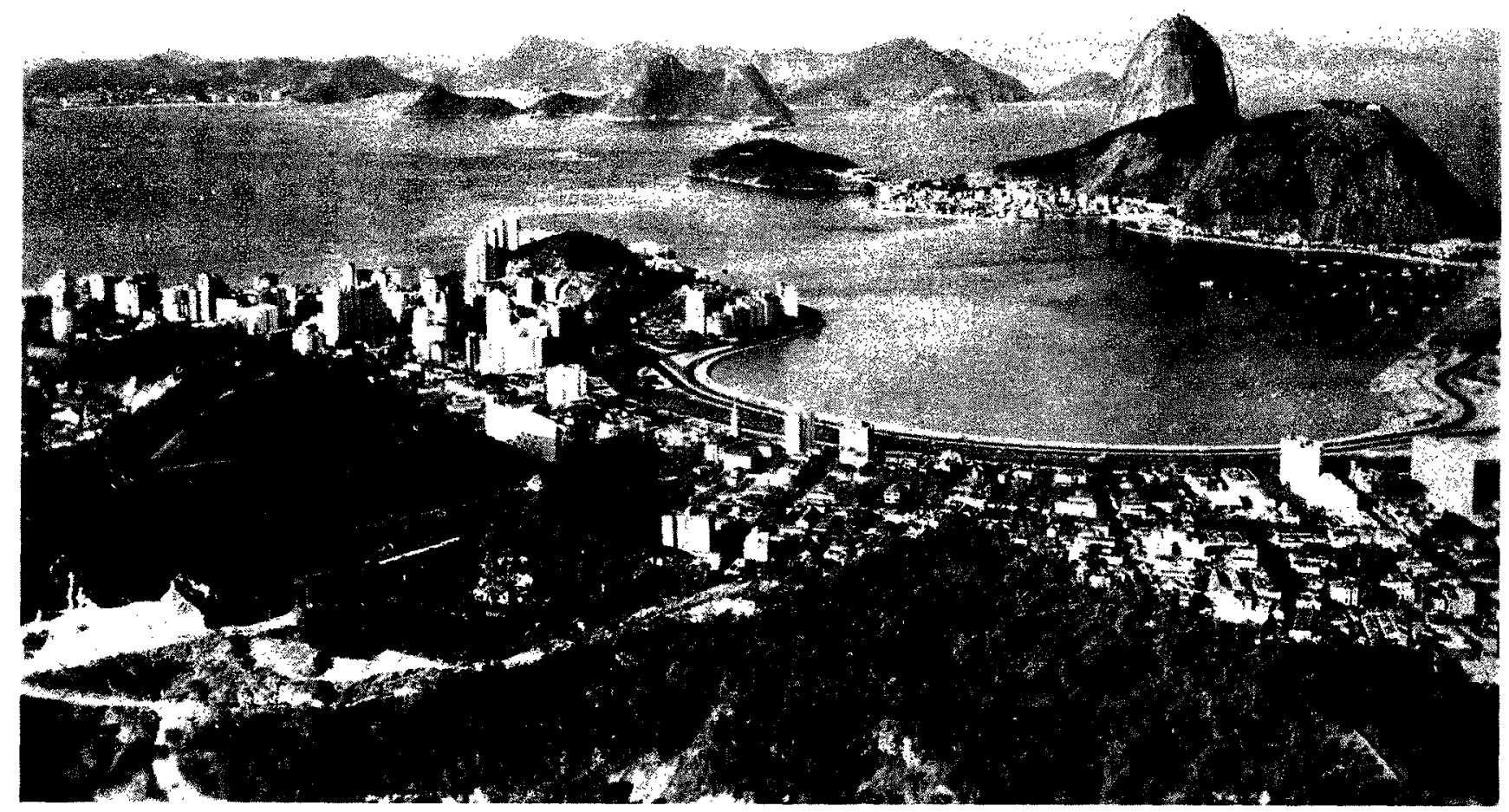

Bate de Rio de JaNerno et he "Pain de SUCre"

Les cites les plus peuplées sont:

Rio de Janeiro... 2.600.000 habitants

São Paulo ...... $2.250 .000 \quad-$

Recife....... $572.000 \quad-$

Salvador. . . . . $420.000 \quad-$

Porto Alegre ... $410.000 \quad \ldots$

Belo Horizonte . . 370.000

Belem. . . . . . $240.000 \quad-$

Santos. . . . . . $210.000 \quad \ldots$

8 a 10 millions seulement d'individus participent i la vie moderne.

Le peuple brésilien est d'une constitution physique plus délicate que l'Européen. La créolisation a cu pour effet une certaine décadence physique. Les maladies les plus fréquentes sont la tuberculose, les maladies vénériennes, la lèpre (15.000 lépreux, 14 préventoria, 24 léproseries) et les maladies du foic, des reins et de l'estomac, ces trois dernières étant beaucoup plus répandues qu'en Furope, sans doute par l'effet d'une alimentation trop échauffante, trop épicée et trop carnée. I.e paludisme y est relativement peu important.

L'indien, au nombre de moins d'un individu au $\mathrm{km}^{2}$ en Amazonie, y souffre lui-même du climat, que lo nègre supporterait mieux. Il est plus sensible que les blancs aux maladies humaines qui n'existaient pas chez eux avant l'arrivée des blancs et qui, béni- gnes chez ceux-ci, premnent chez l'Indien un caractère de gravité catastrophique qui est l'une des raisons pour lesquelles il fuit le plus possible le contact des blancs. Quant au nègre, grand enfant paresseux, doux, émotif, sensible, inconstant, malpropre, incapable d'effort continu, mais prolifique, il résiste assez bien atux maladies.

Le Brésilien est un être complexe, pétri de qualités et de défauts comme tout être humain; il est silencieux, rêveur, sentimental, indolent, insouciant, inconstant, imprécis, inexact, spéculateur et joucur; mais a la fois fier, doux, affable, courtois, hospitalier, complaisant et bienveillant par nature, confiant, serviable avec empressement, un peu formaliste, sensible aux manques d'égards ou de précautions, aux oublis de l'étiquette ou des usages, mais toujours avec donceur et dignité. Grand est son souci de ne jamais blesser en paroles, contravier on refuser; au Brésil, la forme polie de dire non est "a manha ": demain. Cette forme de délicatesse appelle une réciprocité à laquelle le Brésilien est très sensible et très attaché. a $\mathrm{E}$ mais gentil gente »: c'est un peuple très aimable, disait déjà Martim Alfonso de Souza à son arrivée à Rio en 1531 .

\section{Culture}

Au Brésil, la langue parlée est le portugais, mais un portugais brésilianisé quant à l'accent, à l'intonation et parfois aussi à l'expression littéraire, les 


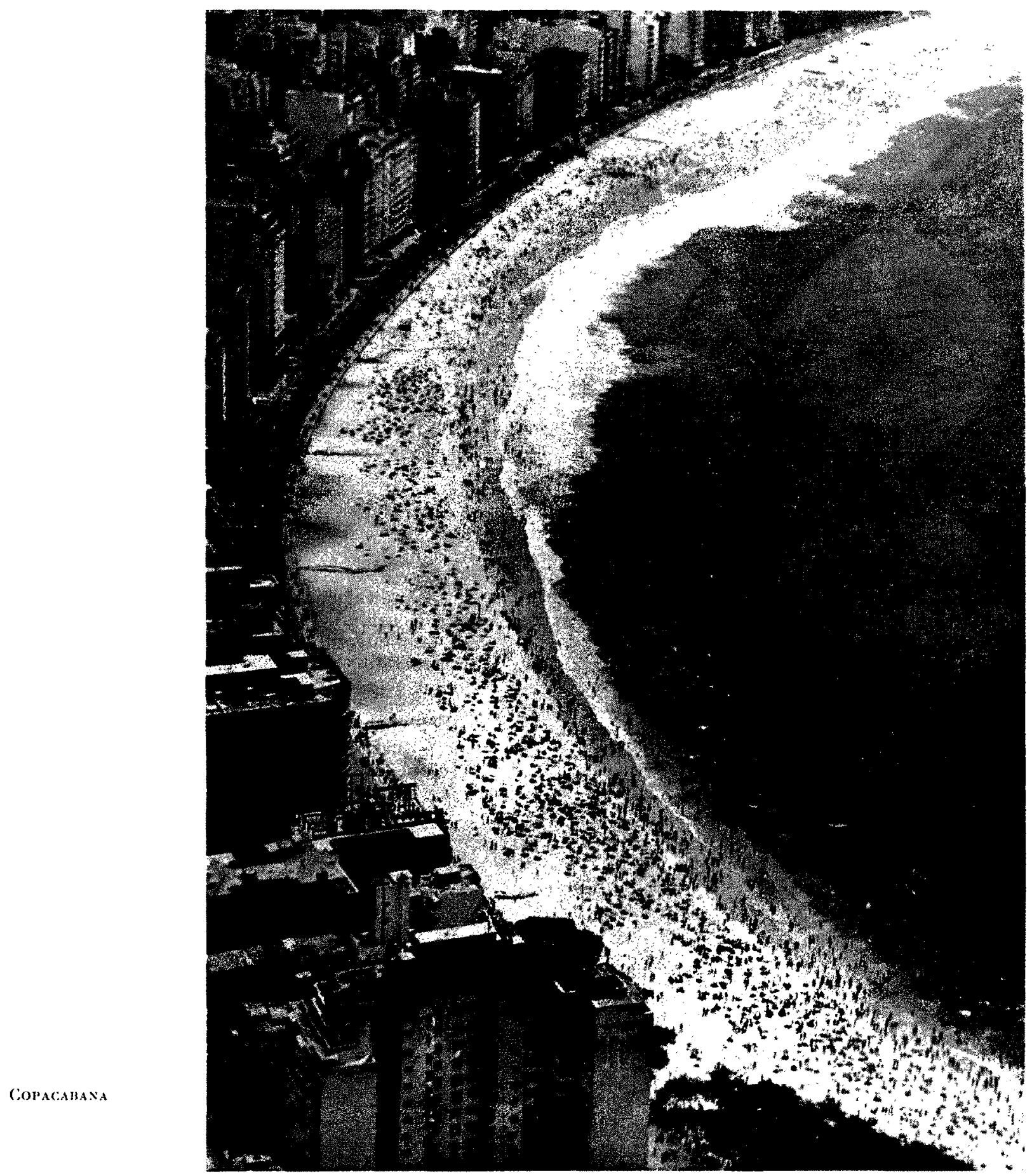

différences pouvant se comparer at colles qui distinguent l'anglais de l'américain.

La religion catholique y est pratiquée par la très grande majorité des habitants.

Une grande partic de la population est encorc analphabète, malgrè les progrès réalisès depuis quelques années: la moitiè seulement des gens âgés de plus de cing ans sait lire et écrire. L'enseignement, de caractère commun ou spécialisé, comprend les degrés primaire, secondaire et supérieur (ce der- nier aree 8 miversites d'litat, 3 minersités catholiques el 1 miversiti rurale) et un enseignement suppletif complementaire el professionnel. On trouve au Brésil plus te 3.000 bibliothèques, dont un millier est spécialise, avec un lotal de 12 millions d'ouvrages; d'importantes archives, plus de 100 musées, de 2.500 periodiques (dont 250 quotidiens et 700 revues), plus de 300 imetteurs de radiodifusion ef de 2.500 salles, tont la plupart sont des cinémas. 


\section{FAMILLE}

La famille forme encore une entile fort vivante au Brésil; e'est encore un véritable groupement forme de deux ou lrois générations, à caractère plus ou moins communatuare, sons l'autorité du pater familias. Le divoree n'est pas admis par la loi bresilienne.

Le grand ennemi de la famille est l'illégitimité des naissances, qui atteint 20 a $50 \%$ dans les Etats du Sud, 50 a $80 \%$ dans le Nord, où les nègres dominent. Beaucoup de naissances ne sont même pass déclarées. La législation a dû reconnaitre des droits successoraux aux enfants nalurels, même adultérins. Par contre, l'adoption ne confère aucun droit successoral aux enfants adoptes, ceci afin de ne pas freiner le magnifique élan d'altruisme qui pousse des familles déjà pourvues d'enfants à adopter de malheureux enfants sans famille.

les villes sont très animées, la circulation et le bruit y sont intenses.

L'agriculture, l'élerage et la sylviculture occupent cnviron $58 \%$ de la population active; l'industric. $y$ compris les industries extractives, et l'artisanat, en occupent environ $10 \%$.

Le standard de vie est très varie; on estime que $33 \%$ de la population totale bénéficie d'un standard de vie analogue an standard enropéen; de ces $33 \%$, win dixième forme la classe aisée, vivant très largement, les neuf autres dixièmes forment la bourgeoisie, allant du boutiquier au petit et moyen fonctionnaire, vivant selon des apparences aisées, mais en réalite assez chichement pour la plupart. Les autres 67 \% sont formés par la masse ouvrière of paysanne, dont la condition est assez misérable, surtout chez les gens de covleur.
La législation sociale, pourtant déjà assez développèe, aura encore beaucoup à faire pour l'amélioration des conditions d'existence de la masse, au regard desquelles les classes dirigeantes agissen avec indifference, ou tout an plus, of individucllement, avec paternalisme. Ie risque social né d'un insuffisant standard de vic doit être envisagé partout ou l'intiative privé ne trouvera pas a sa porlée d'autres secteurs de l'économic on d'autres espáces physiques a mettre en valeur.

\section{Légistation}

Les codes juridiques brésiliens sont un mélange des droits latins de l'Europe et du droit coutumier brifannique. L'habeas corpus y est en vigueur; la peine de mort n'y existe pas (sauf en matière militaire au cours d'hostilités), non pius que le bannissement, la confiscation perpétuelle des biens, la prison pour dettes et l'extradition en matière politique.

\section{Politiole:}

La Constitution en vigueur date de 1946. Le Bresil est une fédération de vingt Ftats, auxquels s'ajoutent un district fédéral (ville et banlieue de Rio de Janeiro) et cing territoires fédéraux (Acre, Amapa, Guaporé, Rio Branco el l'ile de Fernando de Noronha).

Le pouvoir législatif est dévolu a un Sénat de 63 membres et à la Chambre des députes, dus, le premier pour huit ans, et la seconde pour quatre ans, à la représentation proportionnelle, par les Brésiliens des deux sexes àgés de dix-huil ans au moins (les illettrés et ceux ne parlant pas le portugais, exclus). Ie pouvoir exéculit est devolu au presi-

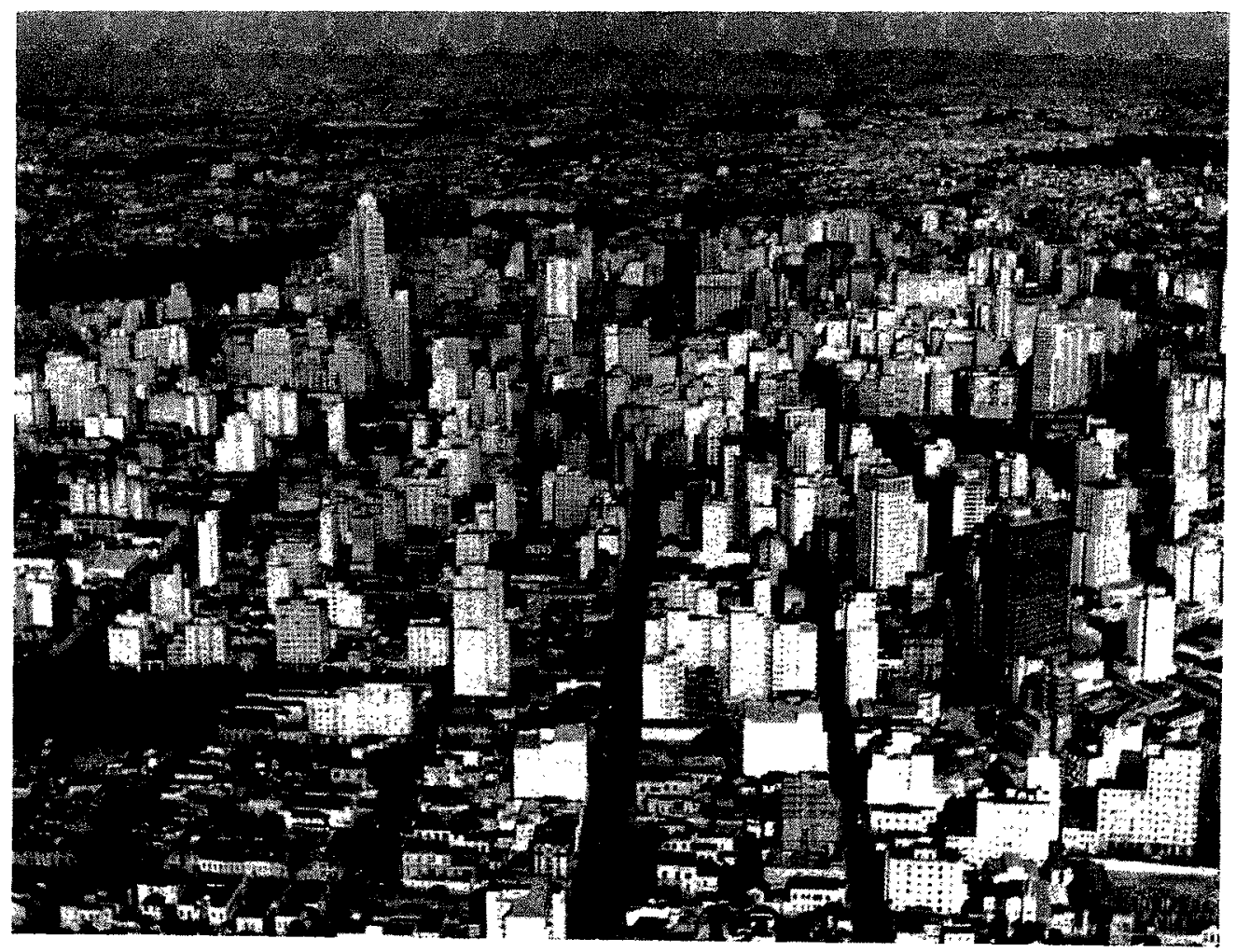


dent de la République, élu pour cinc ans, disposant d'un droit de veto à l'égarl des lois, et dont, en rai son des pouvoirs dont il dispose, laction personnelle peut être très marquée. Le pouvoir judiciaire est représenté, au sommet de la hiérarchie, par la Cour suprême fédérale, composée de 11 membres nommés par le président de la République, avec l'approbation du Sénat.

\section{IMMIGRATION}

La politique démographicque, basée sur des restrictions à l'immigration, inaugurée en 1930 avec le système des quotas, s'est assouplie depuis cette date; la tendance actuelle est, tout en exercant une sélection sévère, de faciliter l'immigration des: étrangers susceptibles d'assimilation.

Les agriculteurs, avant tous autres, sont les bienvenus. Les professions libérales, plus encombrées qu'en Europe, n'offrent guère de débouchés à l'immigration. Les artistes n'y auraient qu'une clientèle restreinte. Les industricls promoteurs d'industries nouvelles, et en général toutes personnes possédant des capitaux susceptibles de s'investir dans l'économie moderne, sont particulièrement bien accueillis.

Les affinités brésiliennes sont dirigées vers le Portugal en premier lieu. L'amitié Iuso-brésilienne est restée très vive : ces deux nations ont tant de motifs d'être fières l'une de l'autre..., et le Brésil conserve à la mère-patric toute son affection; en 1936-1939, lors de la guerre civile espagnole, le Brésil fit savoir qu'il ne tolérerait point une violation éventuelle des frontières portugaises. Encore de nos jouls, les vrais Brésiliens sont tout simplement des « Portugais d'Amérique ». L’influence portugaise est donc et restera l'influence dominante.

Après elle, vient l'inlluence française, restée do beaucoup la plus forte parmi les influences étrangères, mais actuellement surtout intellectuelle et culturelle; le français est la deuxième langue de tout Brésilien cultivé.

\section{III. - ÉCONOMIE}

\section{A) L'économie brésilienne dans le passé}

L'économic brésilienne fut jadis assise durant de longues périodes sur l'exploitation d'un produit unique. On vit ainsi se sucéder six époques, qui portèrent le nom d'un végétal déterminé; nous en resumerons les traits saillants en suivant l'ordre chronologique, mais elles sont caractérisées par deux termes: monoproduction et monopole mondial.

\section{Epoque DU BOIS BRASHL》: 1500-1550 environ}

Depuis la découverte du pays, le pão vermelho, l' « ibirapitinga 》 des indiscenes, fut la seule production exportable. Sa mise en valeur fut l'ouve surtout des «Cristãos Novos », Juifs fraichement convertis, venus du Portugal et d'Espagne et qui, avec les Juifs non baptisés, furent en réalité les seuls colons appartenant à la bourgeoisie; ils deviendront les plus anciennes familles de Babia ou do Pernambouc, les fondateurs du commerce brésilien, et c'est sous leur impulsion que le pão brasil, richesse initiale du pays, lui donnera sa marque économique et son nom.

\section{Epoque de ha CaNne a sucre : 1530-1800.}

S'étant rendu comple que la « Terra dos lapagaios » n'avait pas, comme les Indes, de trésors préexistants à piller et à emporter, qu'il convenait, par suite, de la coloniser et d'y creer des richesses, les Portugais $\mathrm{y}$ importèrent du Cap-Vert la canne à sucre, qui réussit magnifiquement, car elle n'exigeait ni beaucoup de travail, ni grandes connaissances culturales. Les premiers procédés de traitement de la canne, très primitifs, se perfectionnè- rent bientòt et de petites manufactures, les \& engenhos », s'établirent sur les rives de cours d'eau fournissant la force hydraulique. En deux siècles. le Brésil se tailla dans ce domaine une suprématie, un quasi-monopole de fait qui restèrent inattaqués jusque vers 1660 , époque des premières plantations de canne a sucre aux Indesi orientales. Le sucre de canne subit vers 1750 les conséquences de la surproduction et, en 1806, au cours des guerres napoJéoniennes, fut ruiné par l'industrie européenne du sucre de betterave, dont le berlinois Marggraf avait, dès 1747 , décelé l'existence.

Quelques autres productions aidèrent à ce moment au soutien de l'économie brésilienne: le cacao, importé peu après la découverte, l'ćlevage et le tabac; l'importance de ces trois productions était cependant loin d'atteindre celle des précédentes. L'algodon (coton), que les Jésuites apprenaient aux indigènes, dans leurs « aldeias 》 (villages), $\dot{a}$ utiliser et à filer, commençait timidement à jouer le rôle capital qui lui sera dévolu par le xix" siècle.

Notons en passant que la clairvoyance des Jésuites, les incitant à utiliser les éléments locaux, colonisait progressivement l'arrière-pays sur des fondements agricoles; ce développement aboutit $\dot{a}$ une pénétration rayonnante clans toutes les directions. et cela jusqu'à ce que les « bandeirantes », les « mamelucos » paulistes se soient rués sur ces régions prospères pour piller et y razzier des milliers d'esclaves. Réalistes et pensant surtout aux générations futures, les Jésuites furent au Brésil les premiers créateurs de l'idée d'Etat.

\section{Epoque de l'or et du Diamant : 1690-1800.}

Cette période s'ouvre par la découverte de l'or dans la vallée du Rio das Vellıas, dans le Minas 
Geraes, par une petite colonne de Paulistes partis de Taubate pour une «entrada» quelconque. Aussitôt, e'est la ruée, et l'essor inouï de cités-champignons: Villa Rica, Villa Real, Villa Albuquerque, avec 100.000 âmes... $750 \mathrm{t}$ d'or quittent le Brésil pour Lisbonne en un siècle. Mais l'or alluvial finit par s'épuiser, les gites de pierres précieuses par se faire rares, et, après ce siecle de prospérité inoü̈e, la région retombe dans sa situation primitive : Villa Rica, Villa Real, au Inxe insensé et frénétique, cités les plus opulentes et les plus célèbres du Brésil, sont désertées et peu à peu s'annihilent au point qu'on ne les trouve mème plus sur les cartes d'aujourd'hui... Villa Rica, devenue par dérision Villa Pobre, est aujourd'hui Ouro Preto, petite bourgade de province romantique, a la douzaine de ruelles bosselées; a la place de Villa Real est maintenant un pauvre village tapi dans lombre de Belo Horizonte...

Cependant, les laveurs d'or et les «garimpeiros》 (chercheurs de diamant) ont quitté la montagne avec leurs bètes de somme, leurs esclaves et leurs quelques biens meubles et ils ont découvert la plaine fertile. Ils s'y établissent en petites colonies, en petites villes et, peu à peu, une région vide et inculte devient une province active appelée à être l'un des fleurons du pays. Et il en sera ainsi toutes les fois que l'arrêt brutal d'une production stérilisera des cités éphémères: l'exode de leur populalions amène le peuplement et la mise en valeur d'une zone voisine, jadis clésertique ou impénétrable. Illustration de l'adage: à quelque chose malheur est bon.

\section{Epoque du coton.}

Ouverte en réalité depuis fort longtemps - nous l'arons vu plus haut -., elle prend son plein développement à partir de 1768 , quand apparaissent en Europe les métiers à tisser entrainant ce qu'on a appelé la « révolution industrielle ». L'exportation du coton augmenta dans des proportions telles qu'elles exigèrent une plantation et une culture systématiques et organisées, en même temps qu'elles accentuaient l'importation des nègres.

Mais cette production devint peu à peu victime de circonstances défavorables: au-dedans, des sécheresses prolongées, l'abolition de l'esclavage avec la perte de main-d'cuvre quasi gratuite en résultant, la fiève du caoutchouc naissant; audehors, la concurrence nord-américaine depuis la lin de la guerre de Sécession, plus tard celle de i'Egypte. Avec le coton, le Brésil dut commencer à prendre conscience de sa dépendance vis-á-vis de Ia clientèle étrangère, dépendance conditionnée par les prix. Progressivement, le cycle de prépondérance cotonnière brésilien trouva sa fin avant celle du XIX" siècle.

\section{Epoque du caoutchouc: $1840-1910$}

Avant la découverte, quelque 30 millions d'arbres à caoutchouc, Hevea bresiliensis, existaient déjà dans les selvas amazoniennes. Les indigènes en employaient la gomme poisseuse pour calfater leurs pirogues. Quand, en 1839, Charles Goodyear découvre la vulcanisation, le latex noirâtre passe subite- ment au rang des plus grands besoins du monde moderne; l'invention du pneumaticue accroit sa consommation dans des proportions considérables. C'est l'époque des « seringueiros», ces malheureux ouvriers tenus en esclavage et inhumainement exploités par des entrepreneurs sans scrupules. D'autres cités-champignons s'épanouissent oll s'élèvent: Belem, sur la côte; Manaos, en pleine for vierge, cui emportent la palme de la splendeur et du luxe. Le conto de reis vaut alors 200 dollars...

Jusyu'à la fin du $x_{x} x^{\circ}$ siècle, le Brésil réussit à conserver le monopole de la production du latex en prohibant de la façon la plus sévère et la plus jalouse l'exportation des graines ou des plants d'Hevea. Mais un jeune Anglais peut emporter, clandestinement, en Angleterre 70.000 graines, qui sont semées aux Kew Gardens, puis transplantées a Ceylan, à Singapour, aux Indes néerlandaises, enfin en Indochine, où leur culture est soigneusement organisée et où leur exploitation est beaucoup moins onéreuse que dans la brousse amazonienne; en 1910, ils sont en pleine production. La chute est alors vertigineuse : la production brésilienne, de $42.000 \mathrm{t}$ par an en 1910 , tombe à $16.400 \mathrm{t}$ en 1938 , contre $775.000 t$ pour le total des pays précités.

\section{Epoque du caFé.}

A vrai dire, elle remonte à une période bien antérieure à celle du caoutchouc; mais lorsque, vers 1727, la transplantation dans la plaine amazoniemne de quelques buissons de café pris dans la Guyane française ou dans les Antilles eut doté le Brésil de cette nouvelle production, le café était encore un article de luxe et ne pouvait jouer aucun rôle économique. C'est seulement vers 1821 que la consommation européenne et américaine s'intensifie, pour atteindre vers 1850 un niveau très élevé; le Brésil est alors le fournisseur de café du monde entier : entre 1821 et 1940 , il lui en a fourni pour 2 mi]liards de livres sterling. Fatalement, ce caractère de mono-production met le Brésil sous la dépendance des cours du café et sa monnaie, le milreis, en suit les fluctuations. Une surproduction insensée, que facilitent Ia fertilité et l'affinité exceptionnelles de la « terra vermelha », ne tarde pas à avoir des conséquences prévisibles : les cours s'effondrent, et la monnaie les suit. Après de dures mesures d'assainissement du marché, le cycle du café se poursuit de nos jours sur un rythme plus lent.

Jusque très avant dans le $\mathrm{xIx}^{\mathrm{e}}$ siècle, le Brésil fut sous la dépendance économique de l'Angleterre of des autres nations industrielles, succédant $\dot{a}$ lat mainmise régalienne du Portugal; les interdits de Lisbonne, en empèchant pendant trois siècles le Brésil de produire sur son sol beaucoup d'articles nécessaires à son existence : tissus, vêtements, savon, alcool, vin, riz, papier, journaux, livres, " engins », etc..., lui ont fait manquer la révolution industrielle qui, depuis le dernier quart du xyIrre siècle et tout au long du $\mathrm{xix}^{\mathrm{n}}$, a commencé à bouleverser notre monde. Et quand cet élément nouveau : la machine, surgit et fournit dans la journée plus de travail que cent esclaves, l'absence cuasi totale de charbon dans le sous-sol brésilien vient handica- 
per lourdement le pays. Il s'ensuit un retard considérable dans l'évolution des communications, dans le rythme des échanges (en particulier dans le Nord du pays); peu ou pas de voies ferrees sur d'immenses parcours, ni de bateaux à vapeur sur les voies navigables; situation encore aggravée subitement lors de la liberation des esclaves. I'immigration massive d'Européens est alors facilitée pour pallier aux conséquences de cette dernière; col apport de sang blanc éclaircira d'ailleurs visiblement la race brésilienne, que trois siècles d'apports nègres avaient progressivement foncée.

La première guerre mondiale oblige alors le Brésil à s'organiser dans une certaino autarcic. En même temps, la nécessité qu'il y a pour l'économie du pays d'ètre assise sur une pluralitè de produits devient de plus en plus évidente. A défaut des fruits du sol, dont l'ère est révolue, l'industrie apporte ses produits manufacturés et l'on songe déjà aux réserves du sous-sol. En matière agricole, la polyculture se développe aussi. La deuxième guerre mondiale, qui trouve le Brésil mieux préparé que la première, accentue encore la montée de l'industrie, qui s'attaque maintenant à de multiples branches.

\section{B) L'économie brésilienne dans le présent}

\section{AGRICULTURE}

La part de l'agriculture représente encore $1 / 3$ dans le revenu national et les $9 / 10$ dans la valeur des exportations.

La superficie cultivée est d'environ 56 millions d'hectares (2,3\% du total), dont 19 millions en terres et 37 millions en prairies et pâturages permanents. Les forts couvent 395 millions d'hectares, les terres inutilisables, 375 millions; 32 millions d'ha sont susceptibles d'utilisation future. Il ne faut guère songer à mettre en culture plus de 32 millions d'ha supplementaires: les cerratos, les caatingas (savanes a cactées), les pineraies, les régions humides of de climat malsain, les matas (forèts) de régions sèches dites de deuxième qualité, les montagnes, étant pratiquement indéfrichables ou inexploitables. Par ailleurs, les méthodes culturales du passé ont complètement épuisé et détérioré la plupart des terres anciennes du Rio Grande do Sul, du Sáo Paulo et du Pernambouc, qui touchent a la limite de leurs possibilités de production.

Dans l'ordre des superficies cultivées, on classe : maïs, coton, cafe, riz, haricots, manioc, canne it sucre, pommes de terre, blé, bananes.

Le gouvernement soutient les prix de divers pro. duits : coton, riz, haricots, mais, arachide, ble, manioc, maté.

-... Maïs : il occupe près de 5 millions d'ha et rend près de 6 millions de tonnes; en valeur, it represente 12 a $13 \%$ de la production agricole.

- Riz : près de 2 millions d'ha et de 3 millions de: tonnes de paddy; en valeur. $10 \%$ de la production.

- Blé : 810.000 ha et 690.000 tonnes, production qui ne couve que le tiers des besoins du pays.

Haricots : 1.800 .000 ha et 1.150 .000 tonnes.

- Manioc : 1.000 .000 ha et 12 millions de tonnes.

-- Coton : 2.500 .000 ha of 348.000 tonnes de coton en plumes.

-. Ricin (mamona) : 221.000 ha, 158.000 tonnes.

- Catcao : 285.000 ha of 122.000 tonnes.

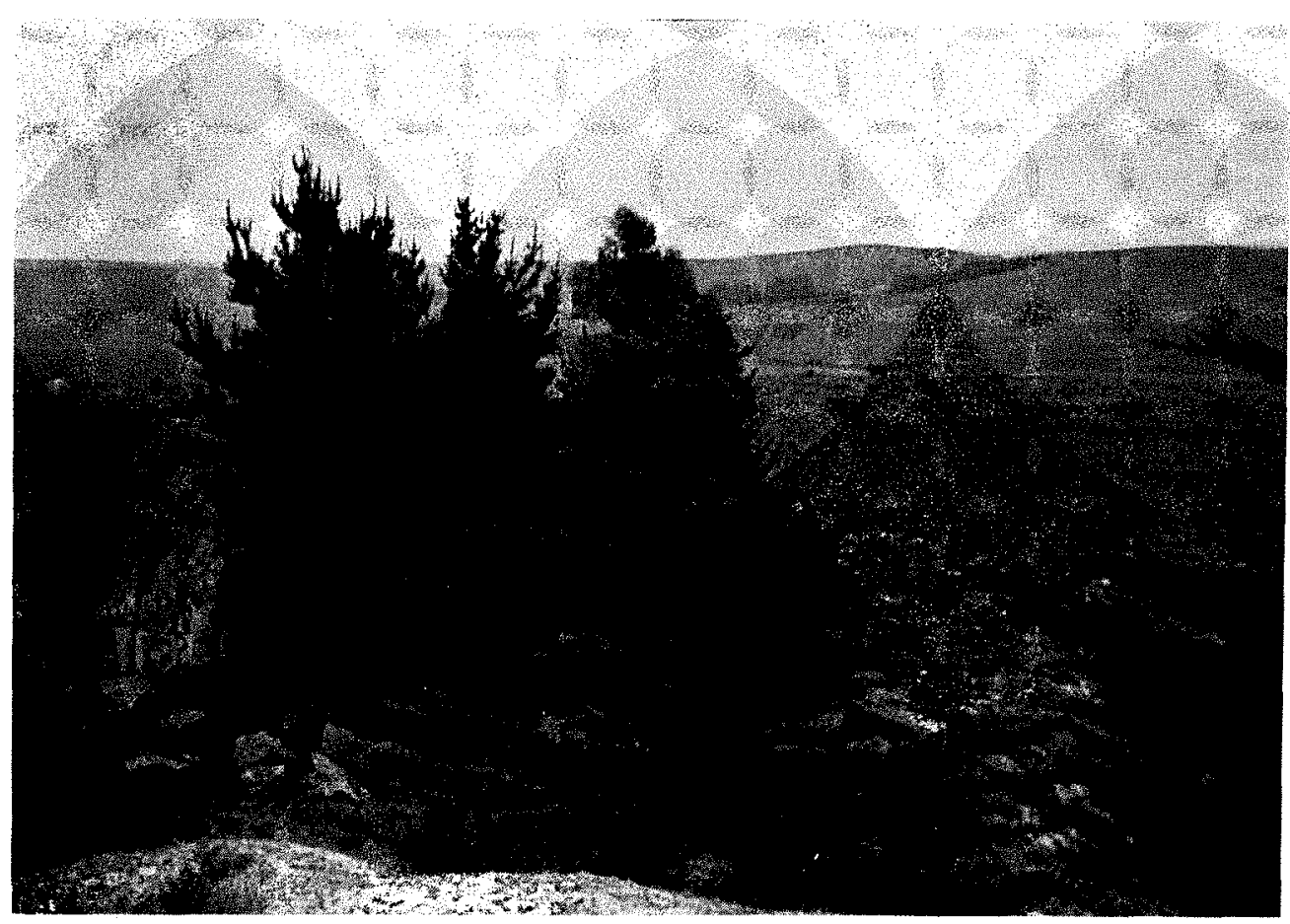




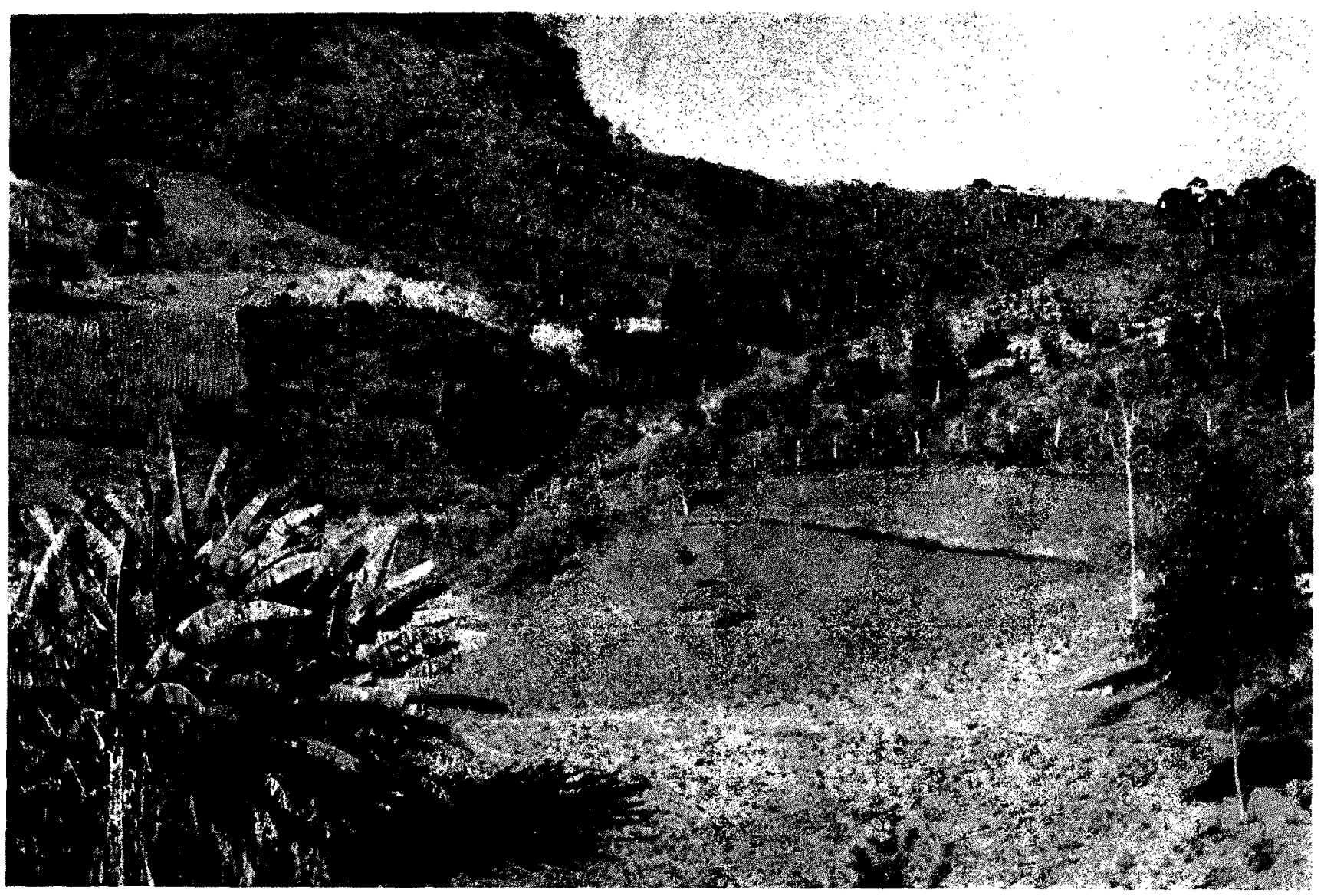

- Café : 2.800.000 ha et 1.120 .000 tonnes; le Brésil reste le plus important producteur de café du monde, avec $45 \%$ de la production mondiale.

-- Les ceréales secondaires, la pomme de terre et la patate donce, l'ail, l'oignon, les tomates, la canne à sucre, l'arachide, les bananes, ananas, abacate, papaye, etc., les agrumes, les cocotiers de Bahia, la vigne et le thé, occupent des superficies parfois importantes et participent intensément à la production agricole.

\section{Elevage}

Bovins... 56.000 .000 têtes (une par habitant) Porcins... $\mathbf{3 1 . 0 0 0 . 0 0 0 ~ - ~}$

Ovins.... 16.000.000 -.

Caprins... $9.000 .000 \quad-$

Chevaux .. $7.500 .000 \quad-\ldots$.

Anes-mulets. $\quad \mathbf{5 . 0 0 0 . 0 0 0 ~}$

La production annuelle de viande atteint 1 million 150.000 tonnes.

Forets. - Couvrant $44 \%$ de la surface du pays, elles sont les plus importantes du monde après celles de l'U.R.S.S. La surface accessible et productrice est de 152 millions d'ha, mais la surface exploite n'est encore que de 40 millions d'ha. Le bois représente 67 à $70 \%$ de l'énergie consomméc au Brésil, l'énergie électrique comptant pour $5 \%$.
Les Heveas produisent annuellement environ 32.000 tonnes de latex. Les autres productions végétales extractives comprennent : les oléagineux : babassu, châtaigne-noix du Para, abrasin, oïticica, murumuru; les plantes a cire : carnaüba, licuri; les plantes à fibres : piassava, caroa, sisal, jute, ramie; les plantes médicinales ou à usages divers : guarana, erva maté, ipeca-cuanha, angico, timbo, corozo.

Pêche. - Le Brésil, malgrè l'étendue de ses côtes et ses $52.000 \mathrm{~km}$ deaux interieures, n'est pas un gros producteur de poisson : 200.000 pêcheurs en capturent seulement $160.000 \mathrm{t}$ par an.

\section{INDUSTRIE}

Depuis la découverte du Brésil, le Portugal y avait prohibé sévèrement toute activité industrielle pour préserver ses monopoles. Quand Dom João VI amorca une politique plus libérale, les traités commerciaux libre-échangistes déjà passés avec l'Angleterre en réduisirent beaucoup la portée. En sorte que le Brésil dut attendre la période commencant en 1880 pour voir son essor industricl se dessiner, grâce aux capitaux brésiliens, d'origine agricole, et étrangers.

La guerre de 1914-1918 accentua cet essor et pris de 6.000 usines furent créées entre 1914 et 1920 ; beaucoup d'entre elles, manquant de bases solides, 
disparurent dans les années qui suivirent. Celles qui subsistèrent furent le berceau des grandes entreprises.

La prédominance industrielle échut rapidement a la ville de São Paulo, favorisée par sa position géographique, son climat salubre dû̀ à son altitude de $800 \mathrm{~m}$, par la facilité de son ravitaillement alimenlaire ansi que des transports ferroviaires ef maritimes (ceux-ci par Santos), par l'abondance ot la qualité de la main-d'œuvre d'origine européenne, et par un bon début d'électrification.

Cependant, après la crise économique de 19291938, et surtout du fait des besoins nés de la deuxième guerre mondiale, une décentralisation accompagna le nouvel essor des industries, principalement de transformation, fonctionnant au départ des matières premières fournies par l'agriculture et l'élevage nationaux, et de demi-produits d'origine? étrangère. Les Etats de Rio de Janeiro, de Rio Grande do Sul, de Minas Geraes, de Pernamboue furent les bénéficiaires de cette décentralisation.

Examinons rapidement la situation présente des divers facteurs intervenant dans l'évolution industrielle:

\section{Main-D'auvre}

Au Brésil, le rendement ouvrier est assez bas; la formation professionnelle à tous les échelons est difficile; au surplus, les cadres supérieurs font souvent défaut, du fait que les étudiants se dirigent plus vers le droit on la médecine -- activités leur permettant plus facilement de s'évader vers la politique -- que vers les sciences; les ingénieurs spécialisés sont rares et beaucoup de postes sont, de ce fait, occupés par des ingénieurs étrangers.

I.es salaires varient énormément, suivant les Ftals, suivant les municipes (departements), limportance des localités, suivant les professions of la specialisation, l'éventail des salaires étant trois on quatre fois plus étali qu'en France. Nous en trouverons les taux au chapitre traitant du coût de la vie.

\section{ENERGLE}

a) Electricité. -... le potentiel hydraulique du pays est évalue a 15 millions de $\mathrm{kW}$, ce qui le place au 4" rang dans le monde, après la liussie, les Etatslinis et le Canata. La puissance installe actuellement est de lordre de $2.250 .000 \mathrm{kVW}$, la production de 10.300 millions de $k W$ et la consommation par tête d'habitant, de $170 \mathrm{~kW}$ par an (contre $1.000 \mathrm{en}$ France). In important programme d'extension de la production d'energie hydraulique est a l'étude. Notons, en passant, que l'orographie du Brésil a permis d'envisager en divers points le systeme de derivation de cours d'eau - .- au besoin par pompage -, pour les acheminer vers des points de chute à très grande dénivellation.

Une dizaine de grandes compagnies, et attant de firmes moyennes, produisent et distribuent l'énergie electrique.

Le gouvernement a envisagé la creation d'une société d'économie mixle, l' «Eletrobras», au capital de 3 milliards de cruzeiros, pour le développement de l'énergie; l'équipement de la chute de Paulo Afonso, sur le Rio São Francisco $(540.000 \mathrm{~kW})$, marque une importante étape dans ce diveloppement.

b) Charbon. -... Le charbon brésilien, peu abondant, est fort médiocre, trop chargé en condres (jusqu'a $43 \%$ ) et en soufre $(6 \%)$, d'un faible pouvoir calorifique $(5.000$ calories a moyenne) et, par

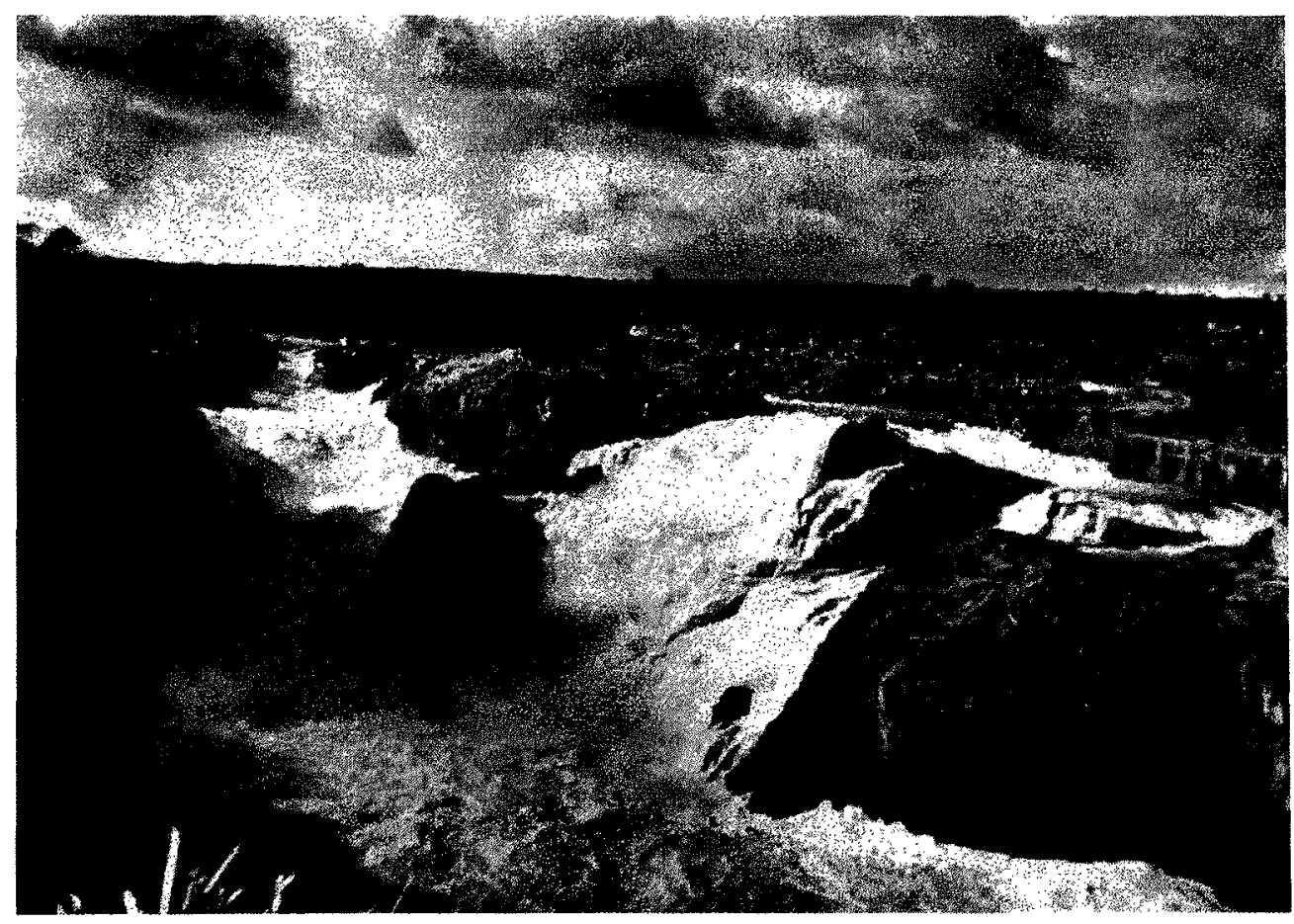


surcroît, trop localisé dans le sud du pays. La production est d'environ 2 millions de tonnes; les importations de charbon étranger de haute qualití sont d'environ $1.280 .000 \mathrm{t}$. Compte tenu de la différence de qualité, des frais d'extraction et de transport, le charbon étranger revient a São Paulo a 20 ou $25 \%$ moins cher que le charbon national.

c) Pétrole. - Les gisements connus sont encore peu importants; la création par le gouvernement de la S. A. "Pétrobras », analogue a l'Eletrobras, mais excluant toute participation etrangèe, ne permettra guère une intensification des recherches, qui exigent une somme de capitatux que le Brésil seul ne peut fournir, et c'est regrettable, etant donnè l'augmentation considérable de la consommation de produits pétroliers, qui dépasse 6 millions de tonnes par an. On ne sait pas encore aujourd'hui s'il y a des réserves importantes de pétrole au Brésil.

L'industrie du rafinage, par contre, est développée, avec sept usines, dont la capacité de raffinage atteint 4 millions de tonnes annuelles.

\section{INDUSTRIES FXTRACTIVIS}

Les prospections et recherches sont encore fort peu avancées si on les compare aux dimensions du territoire, dont les deux tiers n'ont fait l'objet d'aucune tentative.

A part l'or filonien du Minas, le fer, le manganèse et le charbon, les autres gisements sont exploités surtout par environ 60.000 « garimpeiros $\gg$, qui extraient $40 \%$ de l'or total, $90 \%$ du quartz et près de $100 \%$ des pierres précieuses ou connexes; leur productivité individuelle est très faible.

Fer. - Les réserves de minerai a haute teneur sont évaluées à plus de 16 milliards de tonnes, soit près de $22 \%$ des réserves mondiales. La teneur des minerais (hématite) varie entre 55 ef $69 \%$ de fer. Jes principaux gisements sont localises surtont dans le Minas Geraes (serra de Isspinhaco), dans le Matto Grosso, à Urucum, et un peu dans le Bahia, le Ceara, le São Paulo, le Parana et le Sanla Calliarinat.

Ia production est voisine de 2.700 .000 fomnes, dont 1.560 .000 sont exportecs. La consommation intérieure atteint 1.300 .000 tonnes.

Manganèse. - Le Brésil occupe le sixième rang mondial dans la production de ce métal. Les principaux gisements se trouvent à Urucum (Matto Grosso; réserves: 34 millions de tonnes a $45,6 \%$ de $\mathrm{Mn}$ et $11,1 \%$ de $\mathrm{Fe}$ ) et à Macapa (territoire d'Amapa: 25 millions de tonnes). L'extraction annuelle est de 200.000 tonnes.

Banxite. - Réserves totales : 150 millions de tonnes, dont 120 pour le seul gisement de Poco de Caldas (Minas Geraes). Extraction annuelle : 18 a 20.000 tonnes.

Cuivere - Petits gisements très insuffisants.

Plomb. - 450.000 tonnes de minerai à $20 \%$ de plomb. Production : 2.000 tonnes de métal.

Elain. - 18.000 tonnes de cassitérite à $60 \%$ d'étain. Production : 300 tonnes.

Ie chrome, le nickel, l'or, l'argent (insignifiant), le diamant (blanc ou noir), les pierres précieuses ot scmi.précieuses, le quartz, le mica, le tungstène, existent également au Brésil; leurs réserves pernettent de notables exportations.

La mugnésite $\left(\mathrm{CO}^{3} \mathrm{Mg}\right)$ extrêmement pure existe a raison de 750 millions de tonnes dans le Bahia; clle sert à la fabrication des réfractaires.

Pas de gisement de soufre connu; les pyrites sont peu abondantes et l'industrie chimique doit importer $\$ 4.000$ tonnes de soufre par an.

Le Brésil est le seul pays à produire le zirconium, sous forme d'oxyde ou de silicate; on en exporte près de 3.500 tommes, pour environ 2 millions de cruzeiros.

Le titane, le cérium, le thorium, l'arsenic, la fluorine, le tale, la barytine, le cobalt, le lithium, le graphite, le marbre et le sel sont également extraits au Brésil en quantités plus ou moins importantes.

\section{INDISTRIES DE TRANSFOHMATION}

Subirurare. - La production de fonte $(850.0000 \mathrm{t})$, d'acier $(900.0001)$ de de laminés $(750.000 t)$ est loca-

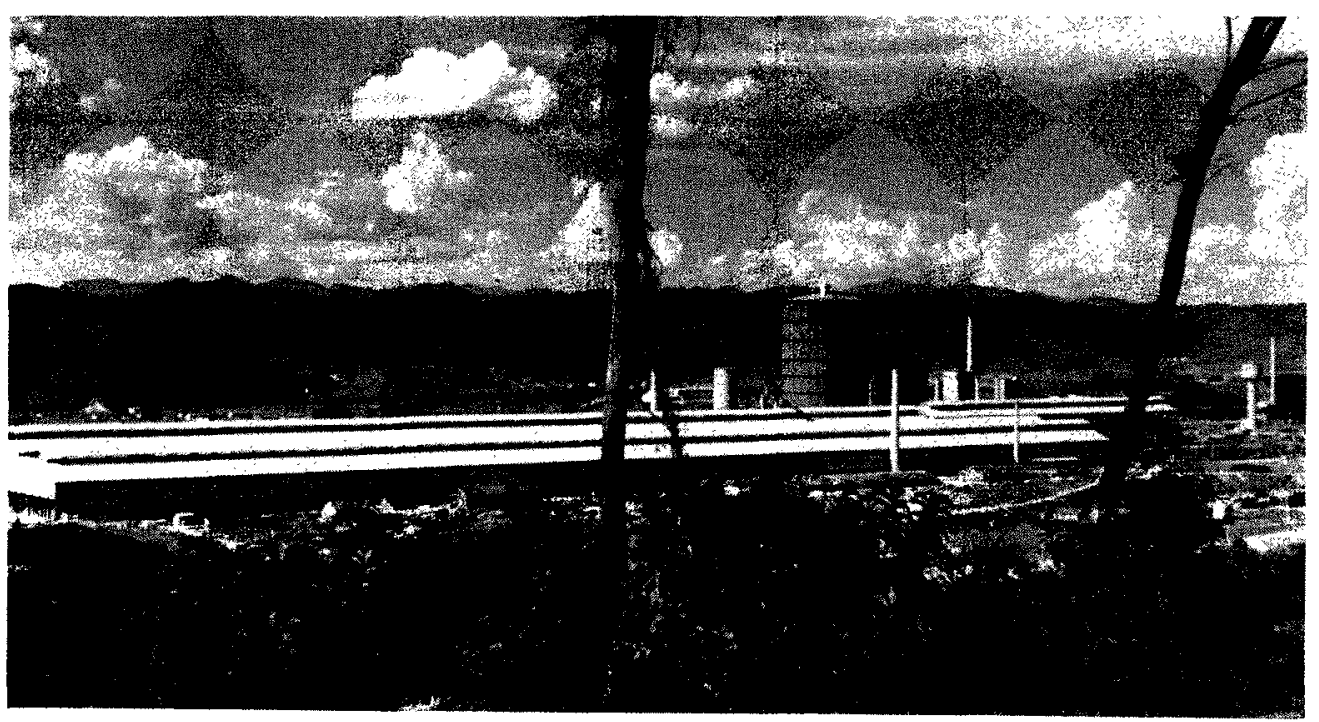

Hall

des laminoirs de la Compiñia Siderurgica Nacional ì

Volta Redonda (São Paulo) 
lisée dans le Minas (9 usines), le Rio de Janeiro (4 usines), parmi lesquelles la plus importante du pays, a Volta Redonda, dans la vallée du Paraiba, pouvant produire prochainement 1 million de tonness d'acier en lingots et $740.000 t$ de laminés, et dans le São Paulo (6 usines).

Aluminium. - Deux usines, dans le Minas; production : $12.000 \mathrm{~L}$, devant passer ultérieurement it $50.000 \mathrm{t}$. La rentabilité de cette production est très discutée. Il y a de multiples usines transformatrices.

Métaux non ferreux. - - Peu importants et nécessitant des importations.

IxDUSTRIES Micasiques. - Elles comprennent :

1. Constructions ferroviaires: une fabrique de locomotives et 5 fabriques de wagons, couvrant plus que les besoins.

2. Automobile : 8 usines se bornent au montage de véhicules importés en pièces détachees; 3 carrosseries et plus de 300 fabriques de pièces détachées. Vraisemblablement, des usines de construction totale seront erées dans un avenir prochain par les Allemands et par les U.S.A., le marché brésilien pouvant absorber 100.000 véhicules par an. Le parc actuel dépasse 650.000 véhicules. Une fabrique d'Etat fait déjà des camions, en collaboration avec une firme italienne.

3. Machines-outils et biens d'équipement: 8 fabriques de machines-ontils et 3 de machines textiles. Les machines à papier, les ascenseurs, les scies, le matériel de levage et de translation, les bétonnières, les concasseurs, le matériel de sucrerie, de distillerie, de chaufferie, etc., sont produits de façon satisfaisante.

4. Divers: La fonderie et l'émaillerie sanitaire, les fourneaux, les armes et munitions, la coutellerie, la quincaillerie, la charpente métallique, la construction électrique, les machines à laver, les frigidaires, les machines à coudre, se développent aussi beaucoup.

INDUSTRE chimique. - Sa production est encore faible : acides minéraux, 200.000 †; alcalis, 43.000 1; carbure de calcium, $25.000 \mathrm{t}$; sulfure de carbone, $6.000 \mathrm{t}$. Elle s'intensifiera par la construction d'usines nouvelles pour l'ammoniaque ef pour les alcalis (Cia Nacional de Alcalis, à Cabo-Frio).

15 sociètés, dont 7 très importantes, et dont 4 sont en relation de filialité avec des firmes francaises, assurent la production ci-dessus mentionnée, celle des engrais phosphatés, des insecticides et des matières plastiques, des pigments (oxydes de zinc, de fer ou de plomb), des silicate, sulfate et sulfure de sorlium, du sulfate d'alumine et des chlorates alcalins. La fabrication du coke, a Volta Redonda, donne annuellement $3.000 \mathrm{~m}^{3}$ de benzol, 580 de toluol, 130 de xylol, 12.000 de goudron brut, 1.000 । de naphtalène, etc.

produits pharmaceatiques: Industrie extremement active, en continuel développement, concentrée à Rio et à São Paulo; 9 firmes principales assurent une production qui couvrira bientôt $80 \%$ des besoins.

Ixuesphe TEXTHE. - 1. Cotonnière : clle com- prend 445 usines, arec 100.000 métiers ef 3.300 .0001 broches, occupant près de 200.0060 personnes.

2. Rayonne: $t$ usines, très modernes (pres de $19.000 \mathrm{t})$.

Les tissages de rayonne et de soie comprennent 18.000 métiers et occupent 25.000 ouvriers.

3. Laine: a) Peignée. 8 grandes filatures, aver 130.000 broches, produisent près de $10.000 \mathrm{t}$ de filés. -b) Cardéc. 5 fllatures, avec 70.000 broches.

Les tissages comprennent près de 50 usines al 7.000 métiers, produisant 15 millions de mères.

4. Lin : 5 firmes, dont une francaise, avec 20.000 broches produisant $2.000 \mathrm{t}$ par an.

5. Jute el fibres dures : 11 usines, avec $3.000 \mathrm{mc}-$ ticrs, abritent 14.000 ouvriers. La production des sacs atteint 60 millions de pieces.

INDLSTREs Diverses. - 13 firmes principales produisent par an $300.000 \mathrm{t}$ de papier; en papier journal, $1 / 3$ seulement des besoins est couvert. 8 fabriques de pneumatiques, 85 de caontchouc industriel, avec 8.000 ourviers, absorbent plus que la produc. tion nationale de latex, d'où l'importation de qualques milliers de tonnes de sruthetique. 8 firmes principales produisent 2 milliards de paquets do cigarettes. La cimenterie (18 firmes, plus 9 en projet), la verreric el la céramique sont bien repre. sentées.

INDUSTRIES ALIMENTARES. - Elles groupent 32.000 établissements, occupant 235.000 personnes, dont la production vaut 34 milliards de er. Parmi eux, on note :

917 moulins, 565 fabriques de pâtes alimentaires, 119 biscuiteries, des conserveries de viandes, de legumes, te fruits et de poisson, 394 sucreries (plus 67.000 « engenhos »), 180 distilleries d'alcool (plus 8.000 « engenhos » produisant l'aguardente), une vinglaine de fabriques d'huile (de coton, ricin, arachide, babassu, lin, oïticica) el de beure de cacao, el 6 grandes savonneries.

Bonssoss. - La biere represente plus de $6 \mathrm{mil}$ lions d'hecholitres. Deux grandes brasseries font aussi le guarana et l'agua-tonica.

la production des vins est faible; elle sol atussi a la fabrication d'apéritifs.

60 millions de litres d'eaux minérales proviennent du Minas, du São Paulo et du District Fédéral.

Plus de 50 firmes francaises ont etabli au Brésil des filiales, dont certaines sont tres importantes.

\section{C) Situation financière}

I'unité monétaire, le milreis (1.000 reis), est appelée depuis 1942 cruzeiro (valant 100 centavos). l.e « conto», ou conto de reis, non officiel, dissigne 1.000 cruzeiros. Selon la Convention du Fonds monetaire international, la parité a été fixée à $18,50 \mathrm{cr}$ pour 1 dollar, ou 5,40 cents pour 1 cruzeiro.

Le change at marche libre est base sur $108 \mathrm{cr}$ pour $1.000 \mathrm{fr}$ français a l'echat ef $114 \mathrm{cr}$ is la vente, soit environ 9,25 a $8,77 \mathrm{fr} /$ pour 1 (ruzeiro. 


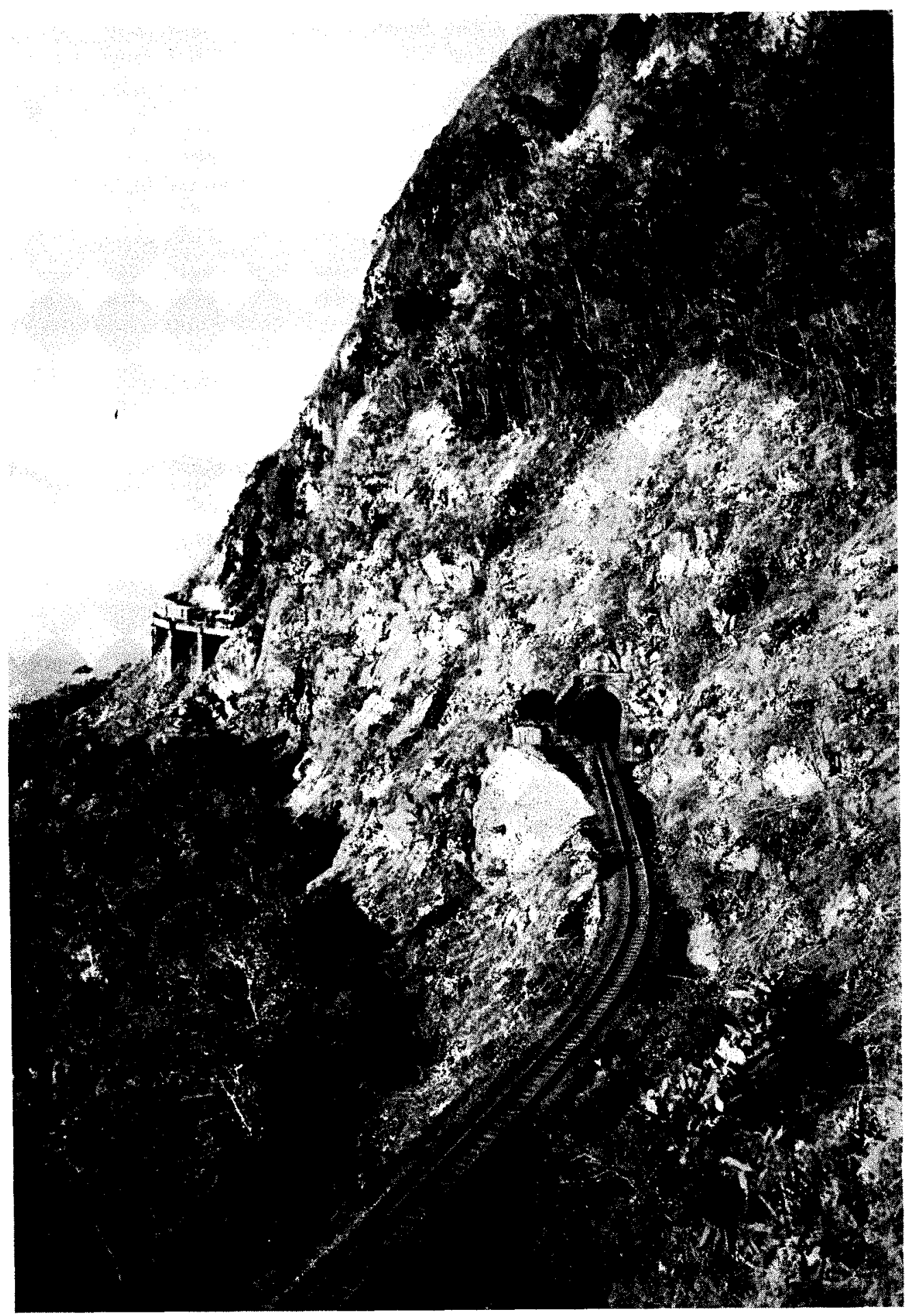

Voie ferede de l'aranagua ì Curityba (Parana)
Le taux de l'intérêt bancaire est elevé :

Escompte commercial .......... de 9 ¿ $12 \%$ Avances garanties et en C. C........ environ $11 \%$

L'intèrêt servi aux dépôts, abaissé depuis 194\$, va de 3 a $4 \%$.

\section{D) Communications}

1. Voles Fenrées. - Elles représentent $37.000 \mathrm{~km}$,
18 lois moins qu'en France; tracées sans plan d'enscmble, avec 3 et meme parfois 5 écartements de voie différents $(0,60 \mathrm{~m}$ à $1,60 \mathrm{~m}$, la voie de $1 \mathrm{~m}$ prédominant), leur matériel est disparate et insuffisant.

Le parc comprend environ 4,000 locomotives et 60.000 wagons à marchandises. Trafic : 10.334 millions de voyageurs-km el 8.487 millions de tonneskm. Il y a 47 entreprises ferroviaires, dont 13 sont importantes. le réseau est concentré surtout dans 


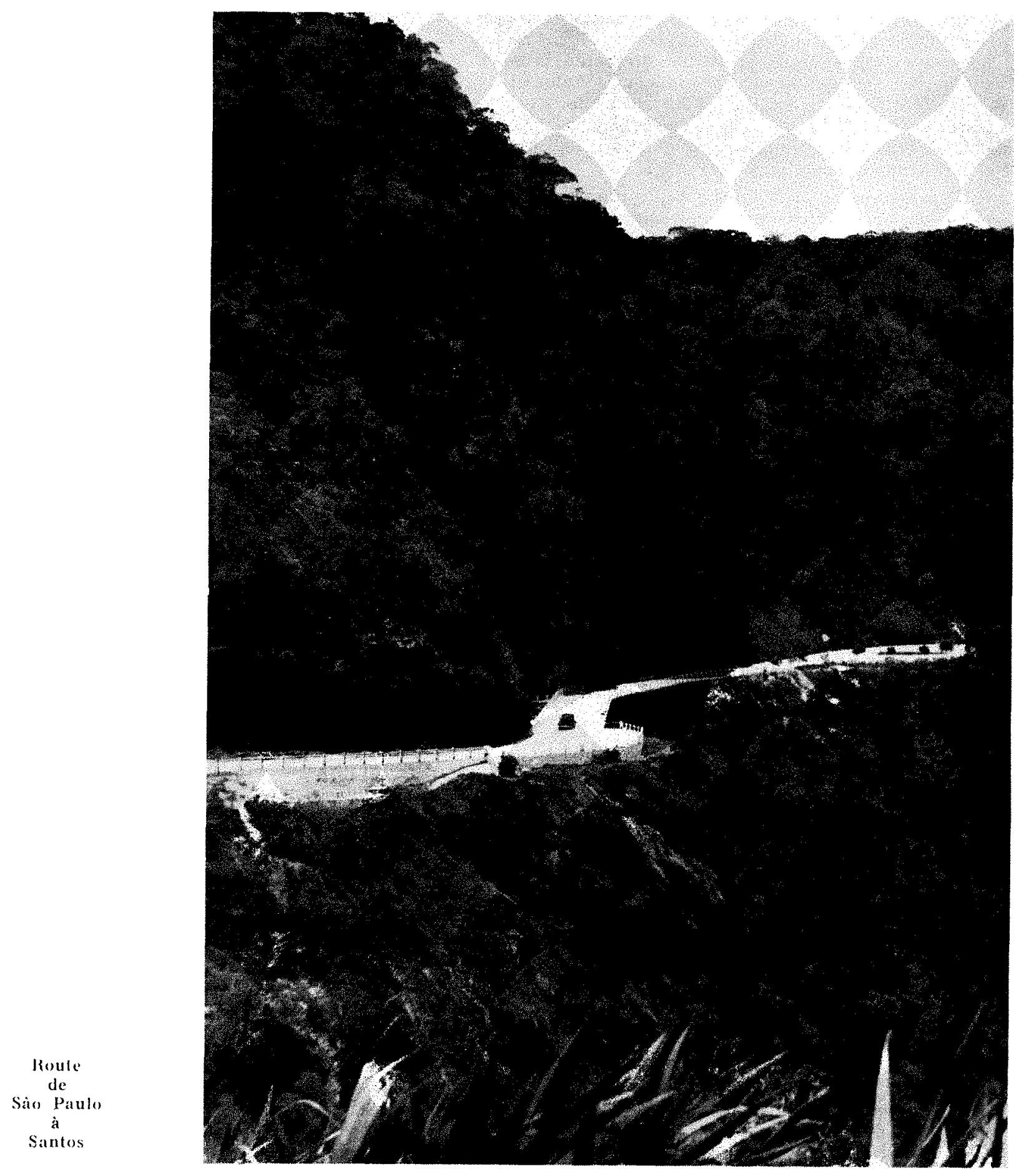

le Minas $(8.672 \mathrm{~km})$, puis dans le São Patto $(7.737 \mathrm{~km})$ et le Rio Grande do Sul $(3.757 \mathrm{~km})$. Ln fonds national a été créé en 1950 pour financer l'équipement.

2. Roures. - Elles représentent $302.000 \mathrm{~km}$, dont $12.000 \mathrm{~km}$ de routes fédérales, $51.000 \mathrm{~km}$ aux Etats, et 239.000 atux municipes.

Ce réseau, insuffisant el de qualité fort irrégulière, comprend plus de $280.000 \mathrm{~km}$ de routes en terre battue, poussiéreuses en périorle sèche, boueuses et impraticables lors des pluies. Les routes du Sud sont les meilleures et celles qui avoisinent les grandes cites sont cimentées ou macadamisées.

3. Traxsports maritimes. - Le cabolage s'exerce sur $6.000 \mathrm{~km}$ de coles. Certains fleuves, nous l'avons vu, sont en partie navigables. On compte 226 ports, dont 21 importants (parmi lesquels Belem et Manaos sont surtout des ports fluviaux), ef 683 petits ports 
lluviaux. Les 6 plus grands ports tolalisent 22 kin de quais, 600 grues, 360 ponts roulants et $525.000 \mathrm{~m}=\mathrm{de}$ magasins. 84 ports totalisent 36.000 entrées de navires, jaugeant au total 50.600.000 tonneaux. Santos et Rio assurent $30 \%$ du total. La flotte marchande est vétuste; en 1950, 106 navires. jaugeant 528.000 tonneaux en brut, appartenaicht a 49 compagnies, dont 2 seules sont importantes.

4. Transponts árress. -..- Le trafic interrieur est presque entièrement réservé aux compaçnies brésilicnnes. Le trafic, en $195^{\circ}$, etait de $100.600 .000 \mathrm{~km}$ (dont 89.400 .000 par compagnies bresiliennes\}, 2.300 .000 voyageurs, $41.310 .000 \mathrm{t}$ de marchandises.

ll y a 8 grandes compagnies, bien subventionnées par i'Etat Férléral; l'aviation privée compte 11.000 pilotes, 2.000 avions, 376 clubs.

5. Traxsports trbarss we conmux. - Les tramways urbains et les 20.000 autocars suburbains sont insuffisants, surtout aux heures d'aflluence, auxquelles d'ailleurs les taxis sont tenus de faire " lotacao $\gg$, c'est-a-dire de prendre en charge des voyaseurs pour une même dircetion, à toutes les places disponibles.

6. P.T.T. - Le trafic achemine 2.216 .000 .000 do leftres, plus 48.000 .000 en service international. 3.057 agences postales renferment 43.402 fonctionnaires; c'est fort insuffisant et le service laisse à désirer. Ie téléphone fonctionne mieux : il comple 550.000 postes, dont 110.000 a São Paulo et 212.000 a Rio; il est assure par 4 compagnies étrangères.

On compte 4.500 .000 récepteurs de T.S.F. ef plus de 300 émetteurs, 2 émetteurs de télévision et 25.000 récepleurs.

\section{E) Commerce}

Commence extrieur. - - Tes importations proviennent des Etats-Lnis $(41,6 \%)$, de l'Allemagne $(9,3 \%)$, de l'Angletere $(8,6 \%)$, de France $(4 \%)$, puis de Suède et de Belgigue.

Les exporlations interessent: les Ftats-Unis (b0 \%), l'Argentine (8\%), la France $(6,8 \%)$, l'Allemagne $(6,7 \%)$, la Suede $(5,3 \%)$, la Hollande $(3,4 \%)$, l'Angleterre $(3,2 \%)$, l'rtalie $(2,75 \%)$, la Belgique ef le Iuxemboura.

las unes ef les autres sont controlees et contingintés.

Commerce intribieur. -- Le commerce de gros des produits agricoles inléresse environ 7.400 négociants (souvent exportateur's également), dont le chiffre d'affaires dépasse 30 milliards de cruzeiros. l.es grossistes et demi-grossistes dans les autres produits atteignent 23.000 unités avec un chiffre de 89 milliards de cruzeiros.

Le commerce de détail groupe 235.000 établissements, pour un chifre d'affaires de 52 milliards de cruzeiros.

\section{F) Coût de la vie. Salaires}

Pour une base 100 en 1946, l'indice était, en novembre 1953, compris entre 211 et 240 , suivant les Etats. Mais la hausse réelle est généralement superieure a celle résultant de ces chifrres. Ia hausse totale entre 1947-1948 et juin 1953 est voisine de $100 \%$.

Les salaires moyens varient, suivant les Etats ef a l'intérieur de ceux-ci, suivant les localités, entre 500 et $1.200 \mathrm{cr}^{\circ}$ par mois.

\section{G) Conclusions}

Les perspectives d'avenir sont, au Brésil, très favorables dans l'agriculture, l'élevage et la production forestière.

Dans l'industrie, il reste une larye marge d'extension, dans la mesure où elle restera protégée et où l'épargne brésilienne consentira à s'y intéresser en délaissant les spéculations immobilières, à rentabilité plus élevée et plus immédiate.

Une stabilisation de la monnaic aurait aussi le plus salutaire effet sur les investissements, surtout émanant de l'étranger.

Le Brésil a son sous-sol encore intact, ses immenses forèts vierges et, de plus, un sol riche qui n'a pas encore été trop détruit par la sottise des hommes et qui fournit, en certains points, deux et même trois récoltes par an (dans le Goias, on vendange en février et en juillet).

Cet immense pays, oil presque lout est encore a faire, depend encore bealucoup de l'etranger pour sa mise en valeur, son industrialisation et sa modernisation. C'est un pays où la vie vaut encore la peine d'ètre vécue pour un homme d'action, ou l'ètre humain n'a pas encore été réduit à la condition d'un termite par les tracasseries gouvernementales et l'ingérence de l'Etat, où existent encore un espace et une libertí - disparues de l'Europe - pour les initiatives individuelles, et qu'il est difficile d'oublier quand on y a séjourné, ce qui explique pourquoi certains tiennent encore pour actuelle l'exclamation d'Amerigo Vespuci quand il y débarqua, mn an après Cabral: "Si le paradis terrestre exisf. quelque part sur la terre, il ne peut être loin d'ici! 》 